\title{
Helix shape power-dependent properties of single upconversion nanoparticles
}

Jiayan Liao ${ }^{1}$, Dayong Jin ${ }^{1,2}$, Chaohao Chen ${ }^{1}$, Yiming $\mathrm{Li}^{3}$, Jiajia Zhou ${ }^{1, *}$

${ }^{1}$ Institute for Biomedical Materials \&Devices (IBMD), Faculty of Science, University of Technology Sydney, Ultimo, New South Wales, Australia

${ }^{2}$ UTS-SUStech Joint Research Centre for Biomedical Materials \& Devices, Department of Biomedical Engineering, Southern University of Science and Technology, Shenzhen, Guangdong, P.R.China

${ }^{3}$ Department of Biomedical Engineering, Southern University of Science and Technology, Shenzhen, 518055, China.

*E-mail: jiajia.zhou@uts.edu.au

\section{Materials and Methods}

\section{Nanoparticle synthesis}

\subsection{Synthesis of $\mathrm{NaYF}_{4}: \mathrm{Yb}^{3+}, \mathrm{Er}^{3+}$ core nanoparticles}

The $\mathrm{NaYF}_{4}: 30 \% \mathrm{Yb}^{3+}, 2 \% \mathrm{Er}^{3+}$ core nanoparticles were synthesized using a coprecipitation method. In a typical procedure, $1 \mathrm{mmol} \mathrm{RECl}_{3}\left(\mathrm{RE}=\mathrm{Y}, \mathrm{Yb}\right.$, Er) with $\mathrm{YCl}_{3} \cdot 6 \mathrm{H}_{2} \mathrm{O}(0.68 \mathrm{mmol}), \mathrm{YbCl}_{3} \cdot 6 \mathrm{H}_{2} \mathrm{O}(0.3 \mathrm{mmol})$ and $\mathrm{ErCl}_{3} \cdot 6 \mathrm{H}_{2} \mathrm{O}(0.02 \mathrm{mmol})$ together with oleic acid $(\mathrm{OA}, 6 \mathrm{~mL})$ and 1-octadecene (ODE, $\left.15 \mathrm{~mL}\right)$ were added to a $50 \mathrm{ml}$ three-neck round-bottom flask under vigorous stirring. The resulting mixture was heated at $150{ }^{\circ} \mathrm{C}$ for 30 mins to form lanthanide oleate complexes. The solution was cooled down to room temperature. Subsequently, a methanol solution (6 mL) containing $\mathrm{NaOH}(2.5 \mathrm{mmol}, 0.1 \mathrm{~g})$ and $\mathrm{NH}_{4} \mathrm{~F}(4 \mathrm{mmol}, 0.14 \mathrm{~g})$ was added and stirred at $50{ }^{\circ} \mathrm{C}$ for $30 \mathrm{mins}$, and then the mixture was slowly heated to $150{ }^{\circ} \mathrm{C}$ and kept for 20 mins under argon flow to remove methanol and residual water. Next, the solution was quickly heated at $300{ }^{\circ} \mathrm{C}$ under argon flow for $1.5 \mathrm{~h}$ before cooling down to room temperature. The resulting core nanoparticles were precipitated by the addition of ethanol, collected by centrifugation at $9000 \mathrm{rpm}$ for $5 \mathrm{~min}$, the final $\mathrm{NaYF}_{4}: \mathrm{Yb}^{3+}, \mathrm{Er}^{3+}$ nanocrystals were redispersed in cyclohexane with $20 \mathrm{mg} / \mathrm{mL}$ concentration after washing with cyclohexane/ethanol/methanol several times.

We synthesized another three kinds of core nanoparticles for similar size with different doping concentrations $\left(\mathrm{NaYF}_{4}: 20 \% \mathrm{Yb}^{3+}, 2 \% \mathrm{Er}^{3+}, \mathrm{NaYF}_{4}: 30 \% \mathrm{Yb}^{3+}, 8 \% \mathrm{Er}^{3+}, \mathrm{NaYF}_{4}: 40 \% \mathrm{Yb}^{3+}, 2 \% \mathrm{Er}^{3+}\right)$ using the same method.

1.2 Synthesis of NaYF4: $5 \% \mathrm{Yb}^{3+}, \mathrm{NaYF}_{4}: \mathrm{x} \% \mathrm{Yb}^{3+}, \mathrm{y} \% \mathrm{Nd}^{3+}(\mathrm{x}=5,15,30 ; \mathrm{y}=20,40,60)$ and $\mathrm{NaYF}_{4}$ pure precursors 
The precursors were prepared as the above procedure until the step where the reaction solution was slowly heated to $150{ }^{\circ} \mathrm{C}$ after adding $\mathrm{NaOH} / \mathrm{NH}_{4} \mathrm{~F}$ solution and kept for $20 \mathrm{~min}$. Instead of further heating to $300{ }^{\circ} \mathrm{C}$ to trigger nanocrystal growth, the solution was cooled down to room temperature to yield the shell precursors.

\subsection{Synthesis of core@ NaYF4:5\% $\mathrm{Yb}^{3+}$ core-shell nanoparticles}

The core@ $\mathrm{NaYF}_{4}: 5 \% \mathrm{Yb}^{3+}$ core-shell nanoparticles were prepared by layer by layer epitaxial growth method. The pre-synthesized $\mathrm{NaYF}_{4}: \mathrm{Yb}^{3+}, \mathrm{Er}^{3+}$ core nanoparticles were used as seeds for shell modification. $0.2 \mathrm{mmol}$ as-prepared core nanocrystals were added to a $50 \mathrm{ml}$ flask containing $3 \mathrm{ml} \mathrm{OA}$ and $8 \mathrm{ml}$ ODE. The mixture was heated to $160{ }^{\circ} \mathrm{C}$ under argon for $30 \mathrm{~min}$, and then further heated to $300{ }^{\circ} \mathrm{C}$. Next, a certain amount of asprepared shell precursors were injected into the reaction mixture and ripened at $300{ }^{\circ} \mathrm{C}$ for $2 \mathrm{~min}$, followed by the same injection and ripening cycles several times to get a shell with $\sim 2.5 \mathrm{~nm}$ thickness. Finally, the slurry was cooled down to room temperature and the formed core-shell nanocrystals were washed and dispersed in cyclohexane for next step epitaxial growth.

1.4 Synthesis of core@NaYF4:5\% Yb ${ }^{3+} @ N a Y F 4: x \% \mathrm{Yb}^{3+}, y \% \mathrm{Nd}^{3+}(x=5,15,30 ; y=20,40,60)$ core-shellshell nanoparticles

The core-shell-shell nanoparticles were prepared by the same epitaxial growth method described above but the core-shell samples were used as the seeds.

1.5 Synthesis of core@NaYF4:5\% Yb ${ }^{3+} @ N \mathrm{NF}_{4}: \mathrm{x} \% \mathrm{Yb}^{3+}, \mathrm{y} \% \mathrm{Nd}^{3+} @ \mathrm{NaYF}_{4}(\mathrm{x}=5,15,30 ; \mathrm{y}=20,40,60)$ core-shell-shell-shell nanoparticles

The core-shell-shell-shell nanoparticles were prepared by the same epitaxial growth method described above but the core-shell-shell samples were used as the seeds.

\section{General materials and optical characterization}

\subsection{TEM and STEM}

The morphology characterization of the nanoparticles was performed by transmission electron microscope (TEM), JEOL TEM-1400 at an acceleration voltage of $120 \mathrm{kV}$ and JEOL TEM-2200FS with the $200 \mathrm{kV}$ voltage. High-angle annular dark field imaging in the scanning TEM (HAADF-STEM) observations were performed on The Helios G4 PFIB series Dual Beam systems with an accelerating voltage of $30 \mathrm{kV}$. The cyclohexane dispersed UCNPs were imaged by dropping them onto carbon-coated copper grids.

\subsection{Absorption spectrum}

The absorption spectrum was measured with the Agilent Cary $60 \mathrm{UV}-\mathrm{Vis}$ spectrophotometer in the wavelength range of 700 to $1100 \mathrm{~nm}$ in a step of $1 \mathrm{~nm}$. 


\subsection{Spectrum detection of ensemble colloidal solution}

The spectral properties of upconversion nanoparticles were measured with a home-built system. A fibercoupled $980 \mathrm{~nm}$ (BL976-PAG500, controller CLD1015, Thorlabs) and $808 \mathrm{~nm}$ fiber laser diode (LE-LS-808300TFCS) diode laser work as the excitation source. The emission spectra of the sample were measured by a commercial spectrometer (Shamrock 193i, Andor) with an EMCCD (iXon Ultra 888, Andor) as the detector. In addition, the emission signal was filtered by a 742 short pass filter (SPF, FF01-750/SP-25, Semrock).

\subsection{Preparation of sample slides for single nanoparticle measurement}

To prepare a sample slide, a cover slip was washed with pure ethanol by ultrasonication, and then the cover slip was air-dried. $20 \mu \mathrm{l}$ of the nanoparticles (diluted to $0.01 \mathrm{mg} / \mathrm{ml}$ in cyclohexane) were dropped onto the surface of cover slip. After being air-dried, the cover slip was put over a clean glass slide before measurement.

\subsection{Confocal single nanoparticle imaging and lifetime measurement}

We built a stage-scan confocal microscope for power-dependent intensity and lifetime measurements of $\mathrm{Nd}^{3+}{ }_{-}$ $\mathrm{Yb}^{3+}-\mathrm{Er}^{3+}$ core-multi shell single nanoparticles, as shown in Supplementary Fig. S2. The excitation source is an $808 \mathrm{~nm}$ single mode polarized laser, which is focused onto the sample through a 100x objective lens (UPLanSApo100X, oil immersion, NA = 1.40, Olympus Inc., JPN). The emission from the sample is collected by the same objective lens and refocused into an optical fiber which has a core size matching with system first Airy disk. The fluorescence signals can be filtered from the laser by a short-pass dichroic mirror (DM, ZT785spxxr-UF1, Chroma Inc., USA) and a short pass filter (SPF, ET750sp-2p8, Chroma Inc., USA). A single-photon counting avalanche photodiode (APD, SPCM-AQR-14-FC, Excelitas Inc., USA) is connected to the collection of multi-mode fiber (MMF, M42L02, Thorlabs Inc., USA) to detect the emission intensity. The scanning is achieved by moving the $3 \mathrm{D}$ piezo stage. During the point by point scanning process, when the excitation laser beam moves closer to a single nanoparticle, the system will detect a brighter emission intensity. Therefore every single nanoparticle will show a Gaussian spot in the confocal scanning microscopic image. The maximum brightness value (photon counts) of each Gaussian spot can be used to represent the brightness of that single particle. For each confocal image, we record the single nanoparticles' brightness values (photon counts). We evaluate more than 30 single nanoparticles' values to calculate the mean brightness.

For the lifetime measurement, we modulate the diode laser to produce $200 \mu$ s excitation pulses. The photon counting SPAD is continuously switched on to capture the long-lifetime luminescence. For each time point, the gate-width is $2 \mu$ s with an accumulation of 10000 times. The pulsed excitation, time-gated data collection and the confocal scanning are controlled and synchronized using a multifunction data acquisition device (USB-6343, National Instruments) and a purpose-built LabVIEW program. 

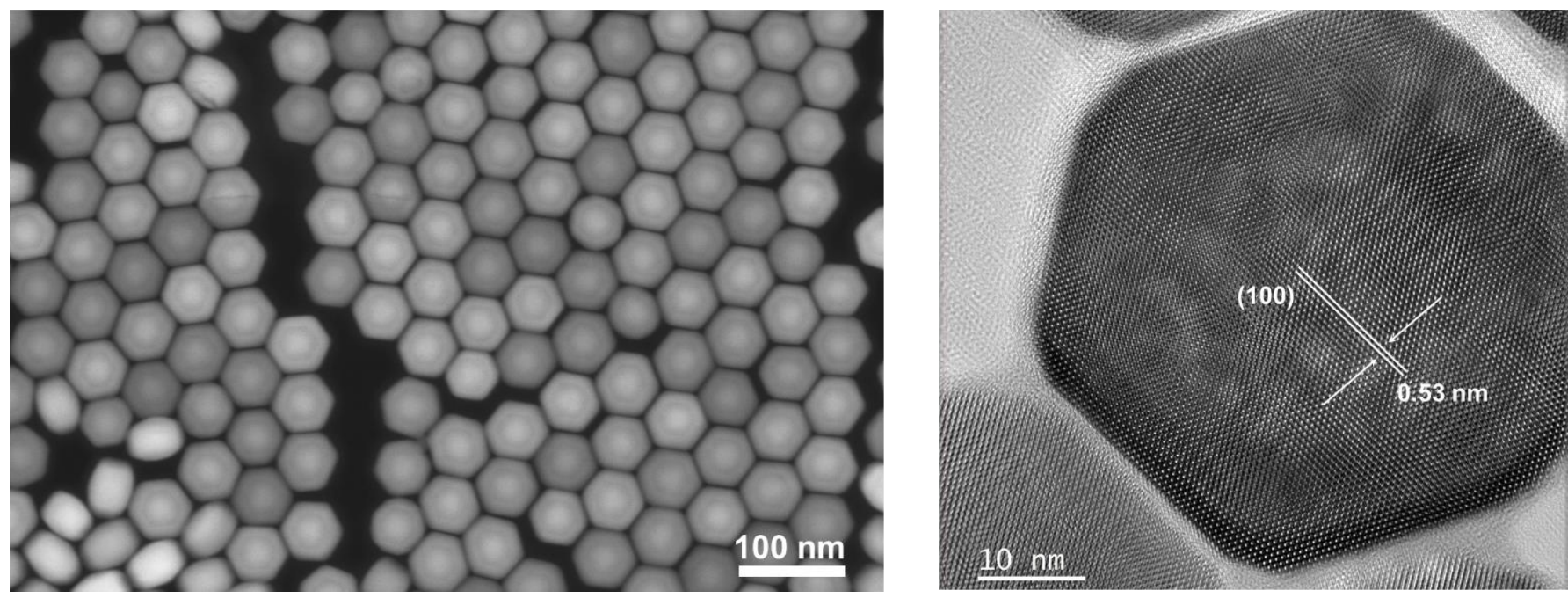

Figure S1. (a) HAADF-STEM image and (b) high-resolution TEM photograph of sample I $\left(\mathrm{NaYF}_{4}: 30 \% \mathrm{Yb}^{3+}\right.$, 2\%:r $\left.{ }^{3+} @ \mathrm{NaYF}_{4}: 5 \% \mathrm{Yb}^{3+} @ \mathrm{NaYF}_{4}: 15 \% \mathrm{Yb}^{3+}, 20 \% \mathrm{Nd}^{3+} @ \mathrm{NaYF}_{4}\right)$. 


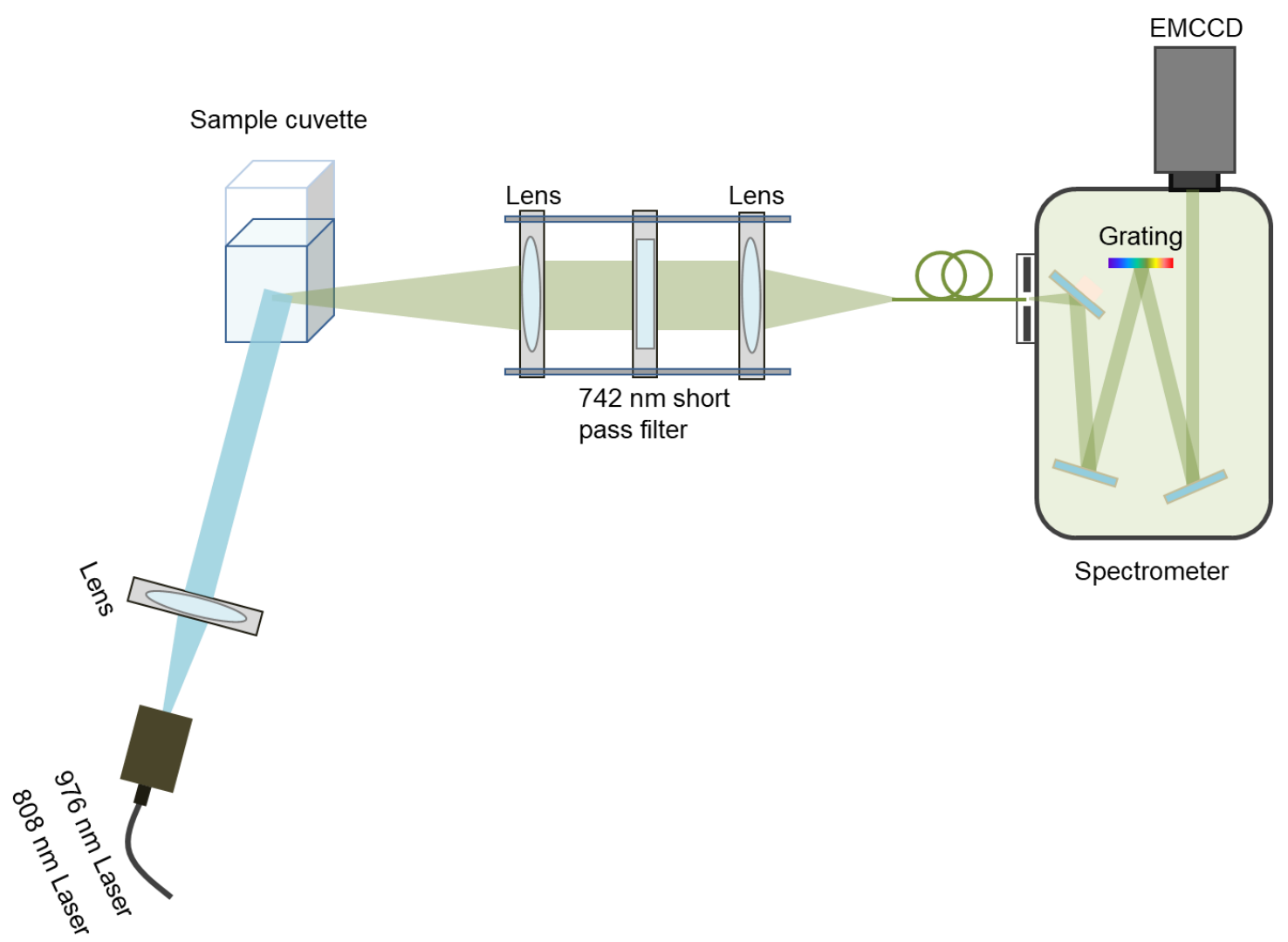

Figure S2. Ensemble spectral measurement setup for upconversion nanoparticles in cyclohexene solution in a quartz cuvette. 


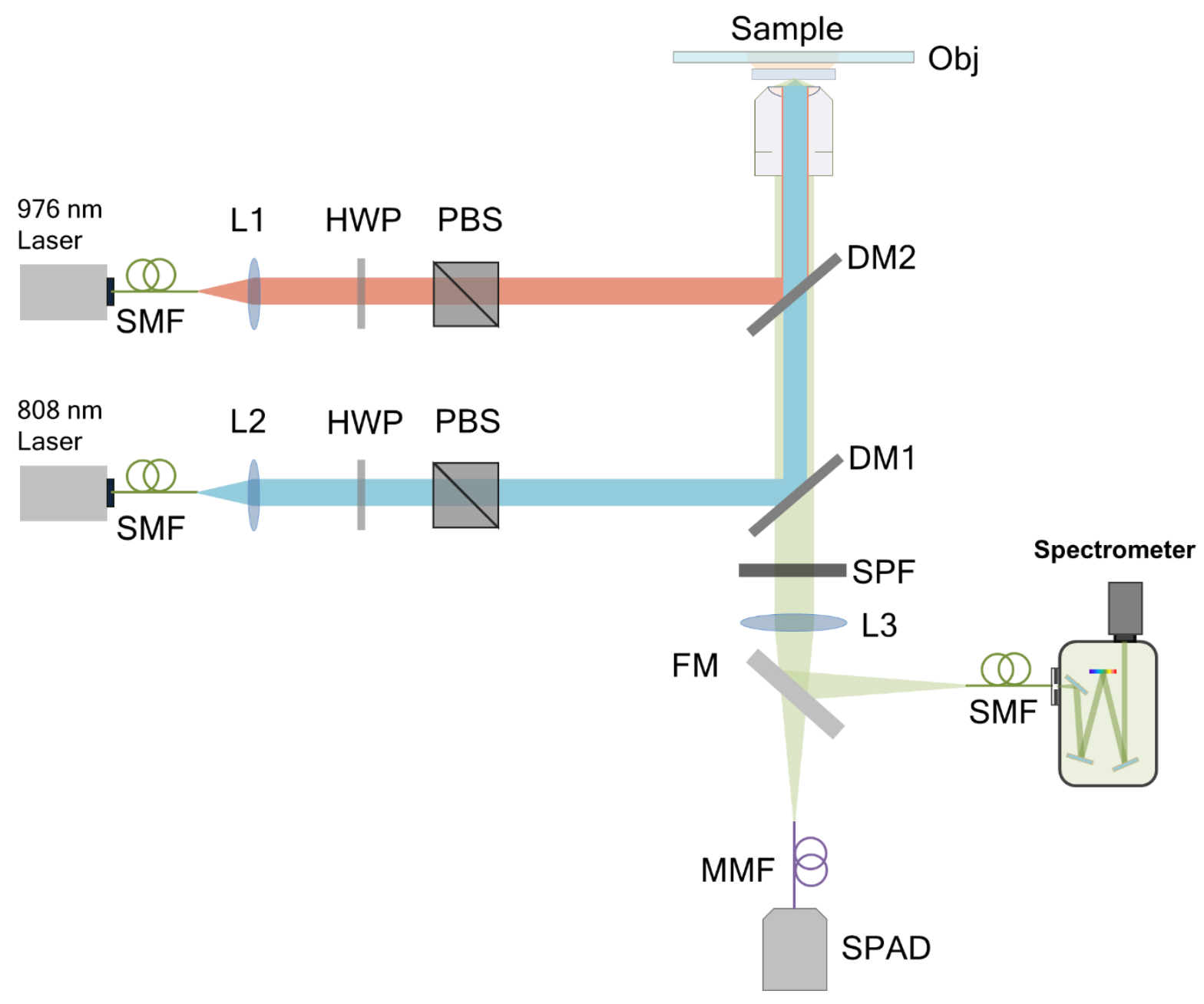

Figure S3. Schematic view of confocal microscopy. (SMF, single-mode fiber; MMF, multi-mode fiber; L1,L2 collimation lens; L3, collection lens; HWP, half-wave plate; PBS, polarized beam splitter; FM, flexible mirror; DM, dichroic mirror; Obj, objective lens; SPF, short pass filter; SPAD, single-photon avalanche diode; Spectrometer (Shamrock 193i, Andor). 

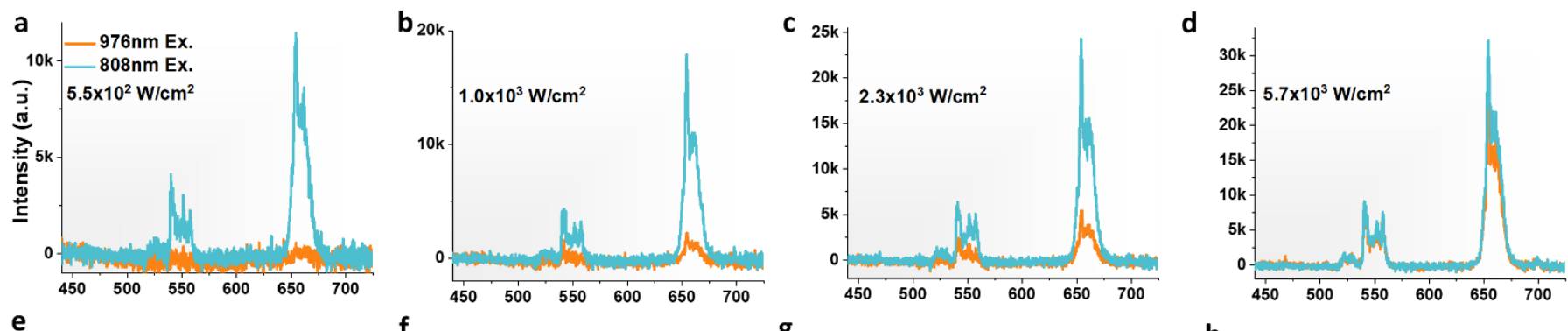

e

f

g
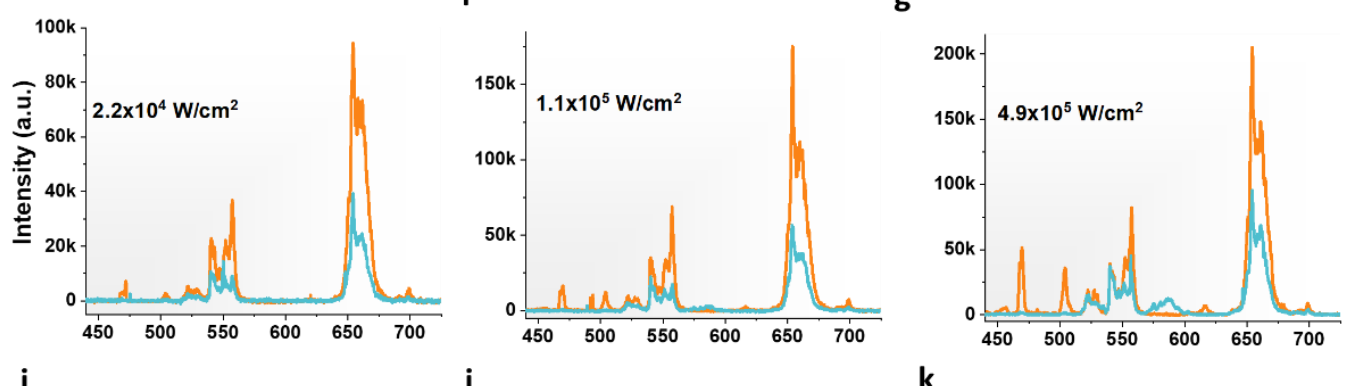

h

i

j
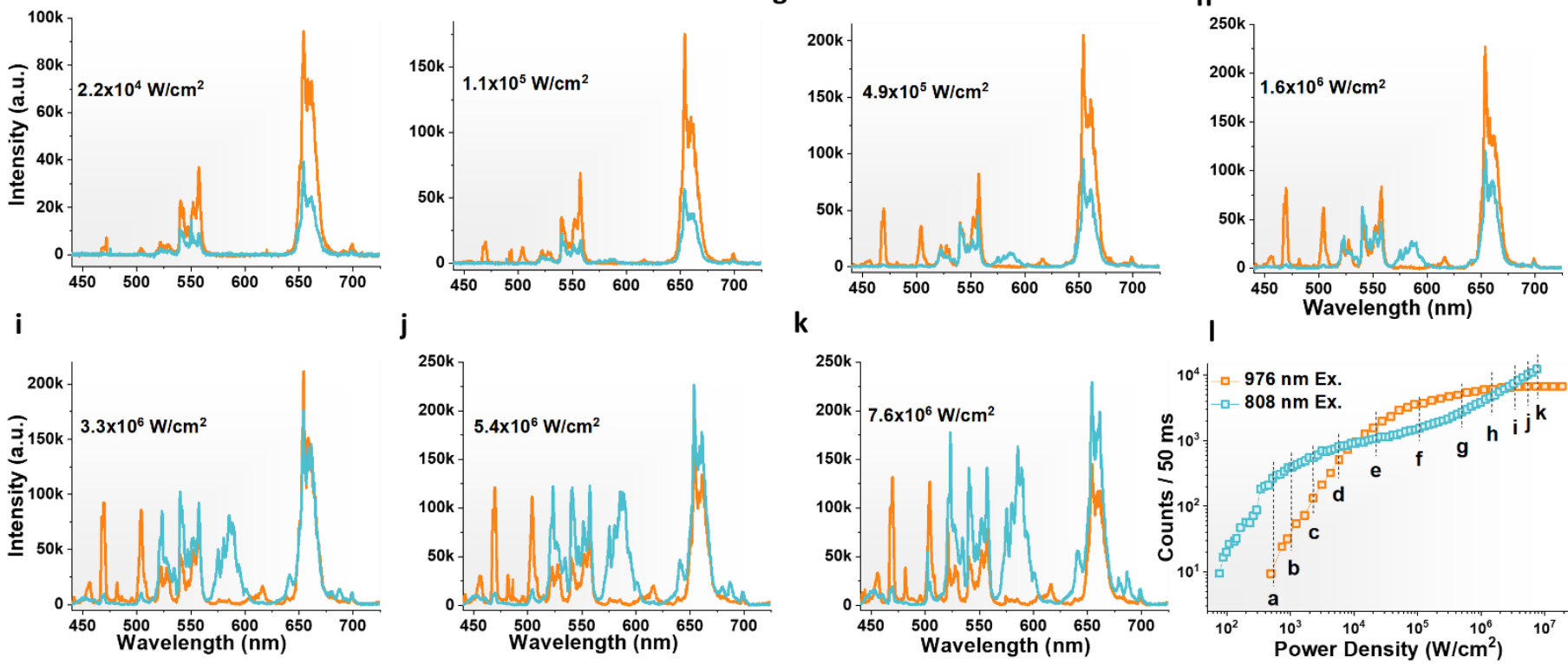

I

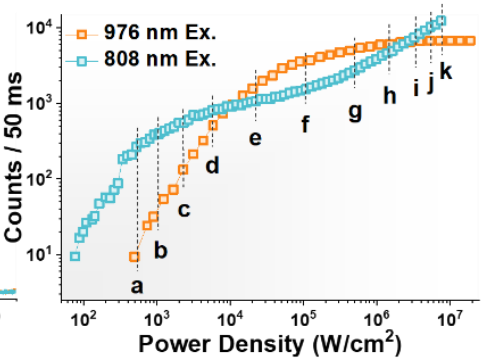

Figure S4. Spectrum variation under $808 \mathrm{~nm}$ excitation source (blue) and $976 \mathrm{~nm}$ excitation source (orange) as the function of excitation power density. 


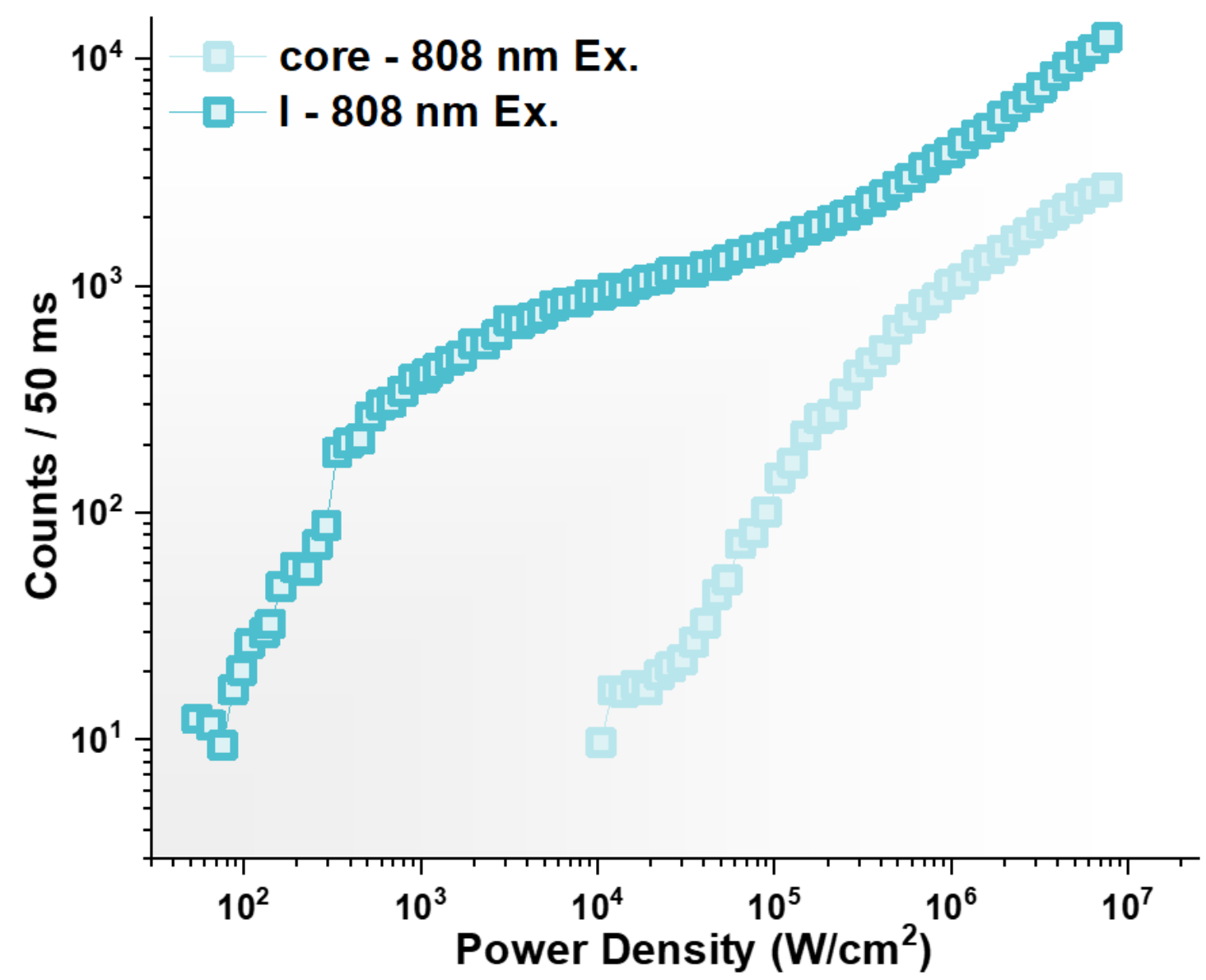

Figure S5. Comparison of the power-dependent brightness between sample I and the corresponding core sample $\left(\mathrm{NaYF}_{4}: 30 \% \mathrm{Yb}^{3+}: 2 \% \mathrm{Er}^{3+}\right)$ at $808 \mathrm{~nm}$ excitation. 


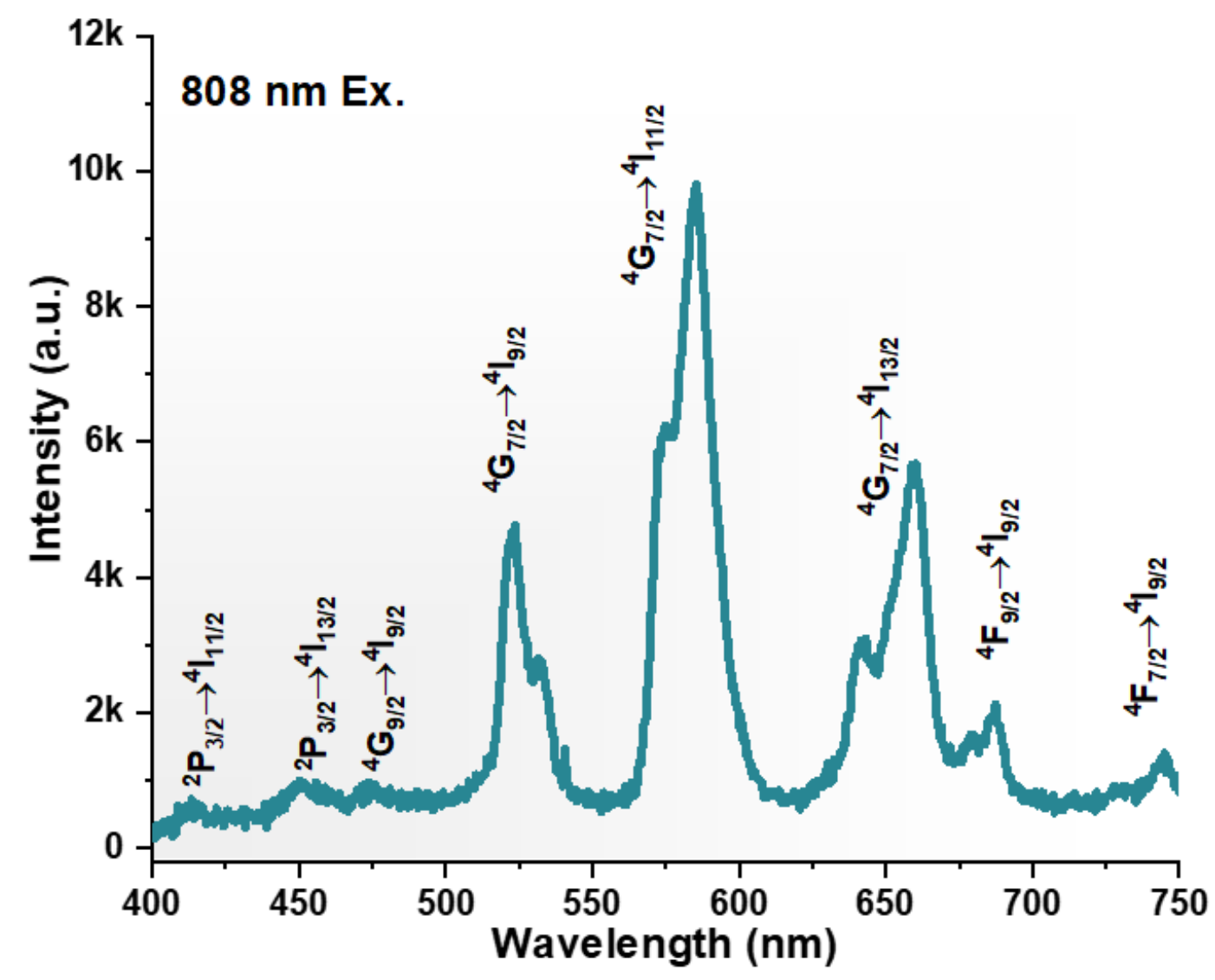

Figure S6. Upconversion emission spectra from $\mathrm{NaYF}_{4}: 15 \% \mathrm{Yb}^{3+}, 20 \% \mathrm{Nd}^{3+}$ single nanoparticle under $808 \mathrm{~nm}$ excitation at $7.6 \mathrm{MW} / \mathrm{cm}^{2}$. 

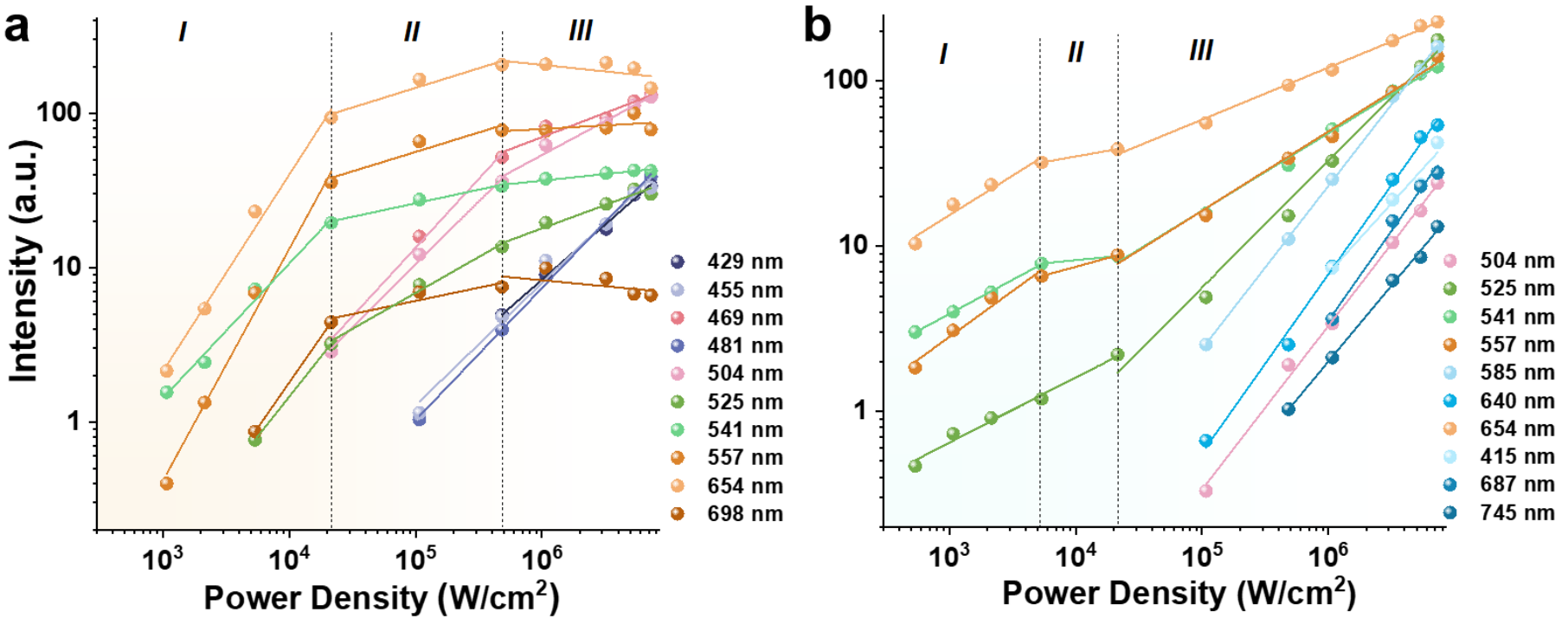

Figure S7. Double logarithmic plots of emission intensities as the function of power density. (a) Power densitydependence of upconversion emission intensity of sample I under $976 \mathrm{~nm}$ excitation at $\mathrm{Er}^{3+}$ emission peaks. (b) Power density-dependent emission intensity changes of sample I under $808 \mathrm{~nm}$ excitation $\mathrm{at}^{3+}$ and $\mathrm{Nd}^{3+}$ emission peaks. The variation tendency of the slope values of $\mathrm{n}$ reveals three phases for the excited state populations. Under these two excitation lasers, the two- and three-photon involved upconversion emissions from $\mathrm{Er}^{3+}$ are observed. Increasing the power density, the populations for the relevant excited states are approaching saturation and thus the slope values decrease. Under $808 \mathrm{~nm}$ excitation in III Phase, the slope values of green and red emission bands of $\mathrm{Er}^{3+}$ ions are bigger than that in II phase because of the superposition of emission bands $\left(525 \mathrm{~nm}:{ }^{4} \mathrm{G}_{7 / 2} \rightarrow{ }^{4} \mathrm{I}_{9 / 2}, 585 \mathrm{~nm}:{ }^{4} \mathrm{G}_{7 / 2} \rightarrow{ }^{4} \mathrm{I}_{11 / 2}, 650 \mathrm{~nm}\right.$ : ${ }^{4} \mathrm{G}_{7 / 2} \rightarrow{ }^{4} \mathrm{I}_{13 / 2}$ ) from direct $\mathrm{Nd}^{3+}$ upconverted luminescence. It indicates the competition of excitation energy flux between energy transfer upconversion of $\mathrm{Nd}^{3+} \rightarrow \mathrm{Yb}^{3+} \rightarrow \mathrm{Er}^{3+}$ and direct upconversion from $\mathrm{Nd}^{3+}$.

Table S1. Summarised slope values for the different emission peaks from single nanoparticle (sample I) in I, II and III Phase under $976 \mathrm{~nm}$ and $808 \mathrm{~nm}$ excitation. While comparing with $976 \mathrm{~nm}$ excitation, the slope values of 2-, 3- photon involved emission peaks under $808 \mathrm{~nm}$ excitation are smaller in I and II Phases.

\begin{tabular}{|c|c|c|c|c|c|c|c|c|c|c|c|c|c|c|c|c|}
\hline Phase & Laser & $\begin{array}{c}413 \\
\mathrm{Nd}^{3+}\end{array}$ & $\begin{array}{l}429 \\
\mathrm{Er}^{3+}\end{array}$ & $\begin{array}{l}455 \\
\mathrm{Er}^{3+}\end{array}$ & $\begin{array}{l}469 \\
\mathrm{Er}^{3+}\end{array}$ & $\begin{array}{l}481 \\
\mathrm{Er}^{3+}\end{array}$ & $\begin{array}{l}504 \\
\text { Er }^{3+}\end{array}$ & 525 & 541 & 557 & $\begin{array}{c}585 \\
\mathrm{Nd}^{3+}\end{array}$ & $\begin{array}{c}640 \\
\mathrm{Nd}^{3+}\end{array}$ & 654 & $\begin{array}{c}687 \\
\mathrm{Nd}^{3+}\end{array}$ & $\begin{array}{c}698 \\
\mathbf{E r}^{3+}\end{array}$ & $\begin{array}{r}745 \\
\mathrm{Nd}^{3+}\end{array}$ \\
\hline \multirow[t]{2}{*}{ I } & 976 & - & - & - & - & - & - & 1.03 & 0.87 & 1.50 & - & - & 1.27 & - & 1.18 & - \\
\hline & 808 & - & - & - & - & - & - & 0.39 & 0.41 & 0.55 & - & - & 0.48 & - & - & - \\
\hline \multirow[t]{2}{*}{ II } & 976 & - & - & - & 0.89 & & 0.82 & 0.47 & 0.18 & 0.25 & - & - & 0.25 & - & 0.17 & - \\
\hline & 808 & - & - & - & - & - & - & 0.39 & 0.07 & 0.21 & - & - & 0.13 & - & - & - \\
\hline \multirow[t]{2}{*}{ III } & 976 & - & 0.71 & 0.79 & 0.31 & 0.86 & 0.44 & $\mathbf{0 . 3 0}$ & 0.08 & 0.047 & - & - & 0.08 & - & 0.077 & - \\
\hline & 808 & 0.85 & - & - & - & - & - & 0.77 & 0.47 & 0.48 & 0.98 & 1.08 & 0.32 & 1.09 & - & 0.92 \\
\hline
\end{tabular}


Table S2. Variation of the decay time of sample I under $808 \mathrm{~nm}$ and $976 \mathrm{~nm}$ excitation sources, in which the laser power density was tuned. (Here, the photon counts located in the wavelength range of $400 \mathrm{~nm}-742 \mathrm{~nm}$ were collected by the single-photon counting avalanche diode. The time constants are defined as the lifetime value corresponding to the two or one fitting coefficient in the exponential fitting. We observe that lifetime value decreases with increasing pump intensity for $\mathrm{Nd}^{3+}-\mathrm{Yb}^{3+}-\mathrm{Er}^{3+}$ doped core-multi shell nanoparticles.

\begin{tabular}{|c|c|c|c|c|}
\hline \multirow[b]{2}{*}{ Power density } & \multicolumn{2}{|c|}{976 nm Excitation } & \multicolumn{2}{|c|}{$808 \mathrm{~nm}$ Excitation } \\
\hline & t1 & t2 & t1 & t2 \\
\hline $5.7 \mathrm{KW} / \mathrm{cm}^{2}$ & $337.62 \mu \mathrm{s}$ & & $832.88 \mu \mathrm{s}$ & \\
\hline $11 \mathrm{KW} / \mathrm{cm}^{2}$ & $282.31 \mu \mathrm{s}$ & $591.69 \mu \mathrm{s}$ & $686.98 \mu \mathrm{s}$ & \\
\hline $22 \mathrm{KW} / \mathrm{cm}^{2}$ & $168.63 \mu \mathrm{s}$ & $401.99 \mu \mathrm{s}$ & $728.19 \mu \mathrm{s}$ & \\
\hline $54 \mathrm{KW} / \mathrm{cm}^{2}$ & $107.55 \mu \mathrm{s}$ & $349.99 \mu \mathrm{s}$ & $753.17 \mu \mathrm{s}$ & \\
\hline $110 \mathrm{KW} / \mathrm{cm}^{2}$ & $82.73 \mu \mathrm{s}$ & $344.59 \mu \mathrm{s}$ & $673.66 \mu \mathrm{s}$ & \\
\hline $490 \mathrm{KW} / \mathrm{cm}^{2}$ & $59.28 \mu \mathrm{s}$ & $329.66 \mu \mathrm{s}$ & $353.30 \mu \mathrm{s}$ & $677.6 \mu \mathrm{s}$ \\
\hline $1.1 \mathrm{MW} / \mathrm{cm}^{2}$ & $52.18 \mu \mathrm{s}$ & $316.51 \mu \mathrm{s}$ & $181.41 \mu \mathrm{s}$ & $592.25 \mu \mathrm{s}$ \\
\hline 3.3 MW/cm² & $39.15 \mu \mathrm{s}$ & $282.6 \mu \mathrm{s}$ & $107.71 \mu \mathrm{s}$ & $439.08 \mu \mathrm{s}$ \\
\hline 7.6 MW/cm² & $31.13 \mu \mathrm{s}$ & $256.61 \mu \mathrm{s}$ & $81.36 \mu \mathrm{s}$ & $385.54 \mu \mathrm{s}$ \\
\hline
\end{tabular}




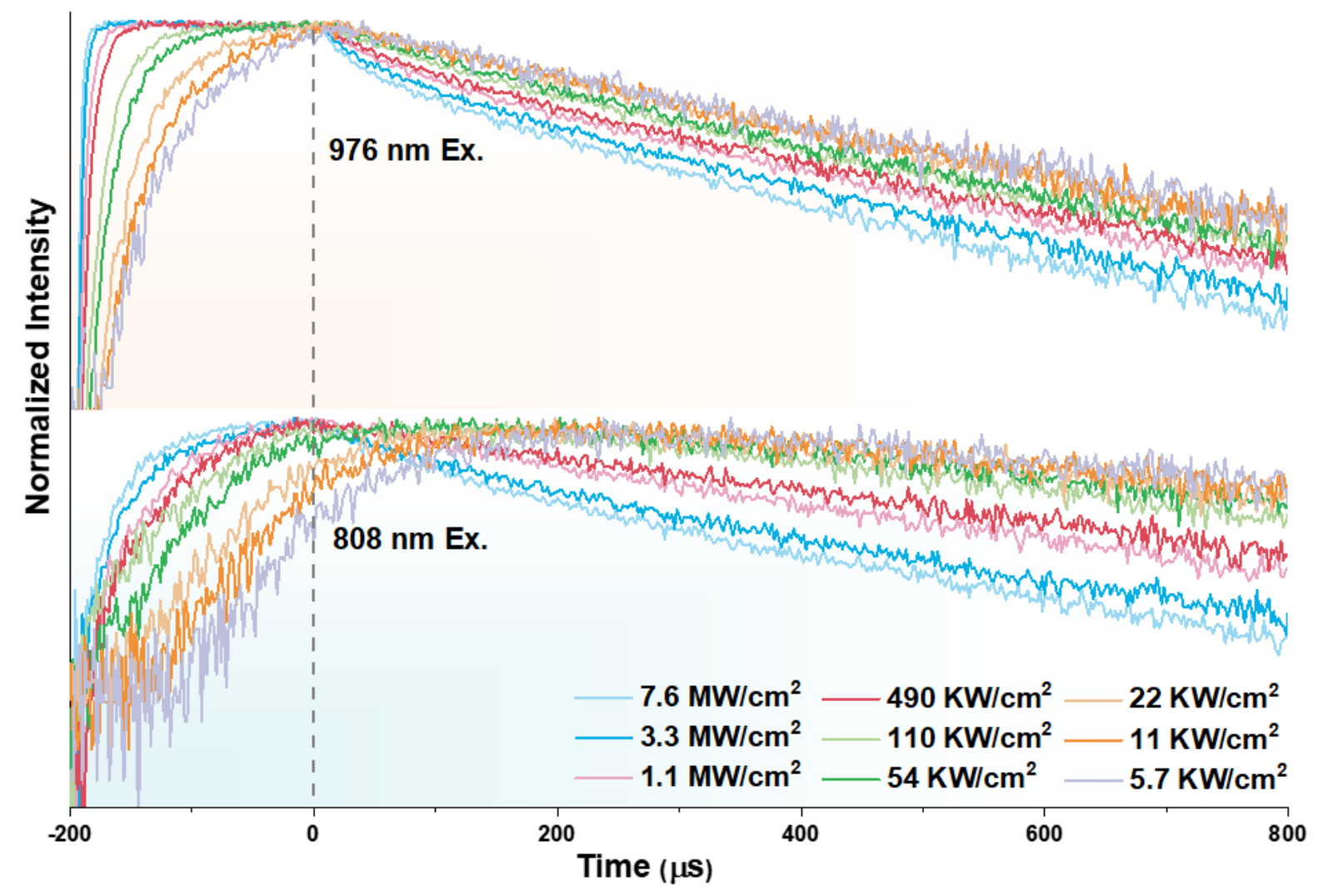

Figure S8. The power density-dependent single nanoparticle lifetime curves, which correlate to the emission signal from the wavelengths between $400 \mathrm{~nm}$ and $742 \mathrm{~nm}$, under $976 \mathrm{~nm}$ (up) and $808 \mathrm{~nm}$ (down) excitation with $200 \mu \mathrm{s}$ pulse width. 
Table S3. Summary of the composition of core-multi shell UCNPs.

\begin{tabular}{|c|c|c|c|c|c|}
\hline & Sample & core & $\begin{array}{l}\text { Migration } \\
\text { Layer }\end{array}$ & $\begin{array}{l}\text { Sensitization } \\
\text { Layer }\end{array}$ & Inert Layer \\
\hline \multirow[t]{2}{*}{$\mathrm{Er}^{3+}$ in core } & I & $30 \mathrm{Yb}^{3+} / 2 \mathrm{Er}^{3+}$ & $5 \mathrm{Yb}^{3+}$ & $15 \mathrm{Yb}^{3+} / 20 \mathrm{Nd}^{3+}$ & $\mathrm{Y}^{3+}$ \\
\hline & II & $30 \mathrm{Yb}^{3+} / 8 \mathrm{Er}^{3+}$ & $5 \mathrm{Yb}^{3+}$ & $15 \mathrm{Yb}^{3+} / 20^{3+} \mathrm{Nd}$ & $\mathrm{Y}^{3+}$ \\
\hline \multirow[t]{2}{*}{$\mathrm{Yb}^{3+}$ in core } & III & $20 \mathrm{Yb}^{3+} / 2 \mathrm{Er}^{3+}$ & $5 \mathrm{Yb}^{3+}$ & $15 \mathrm{Yb}^{3+} / 20 \mathrm{Nd}^{3+}$ & $\mathrm{Y}^{3+}$ \\
\hline & IV & $40 \mathrm{Yb}^{3+} / 2 \mathrm{Er}^{3+}$ & $5 \mathrm{Yb}^{3+}$ & $15 \mathrm{Yb}^{3+} / 20 \mathrm{Nd}^{3+}$ & $\mathrm{Y}^{3+}$ \\
\hline \multirow{2}{*}{$\begin{array}{c}\mathrm{Yb}^{3+} \text { in Sensitization } \\
\text { Layer }\end{array}$} & $\mathrm{V}$ & $30 \mathrm{Yb}^{3+} / 2 \mathrm{Er}^{3+}$ & $5 \mathrm{Yb}^{3+}$ & $5 \mathrm{Yb}^{3+} / 20 \mathrm{Nd}^{3+}$ & $\mathrm{Y}^{3+}$ \\
\hline & VI & $30 \mathrm{Yb}^{3+} / 2 \mathrm{Er}^{3+}$ & $5 \mathrm{Yb}^{3+}$ & $30 \mathrm{Yb}^{3+} / 20 \mathrm{Nd}^{3+}$ & $\mathrm{Y}^{3+}$ \\
\hline \multirow{2}{*}{$\begin{array}{c}\mathrm{Nd}^{3+} \text { in Sensitization } \\
\text { Layer }\end{array}$} & VII & $30 \mathrm{Yb}^{3+} / 2 \mathrm{Er}^{3+}$ & $5 \mathrm{Yb}^{3+}$ & $15 \mathrm{Yb}^{3+} / 40 \mathrm{Nd}^{3+}$ & $\mathrm{Y}^{3+}$ \\
\hline & VIII & $30 \mathrm{Yb}^{3+} / 2 \mathrm{Er}^{3+}$ & $5 \mathrm{Yb}^{3+}$ & $15 \mathrm{Yb}^{3+} / 60 \mathrm{Nd}^{3+}$ & $\mathrm{Y}^{3+}$ \\
\hline
\end{tabular}



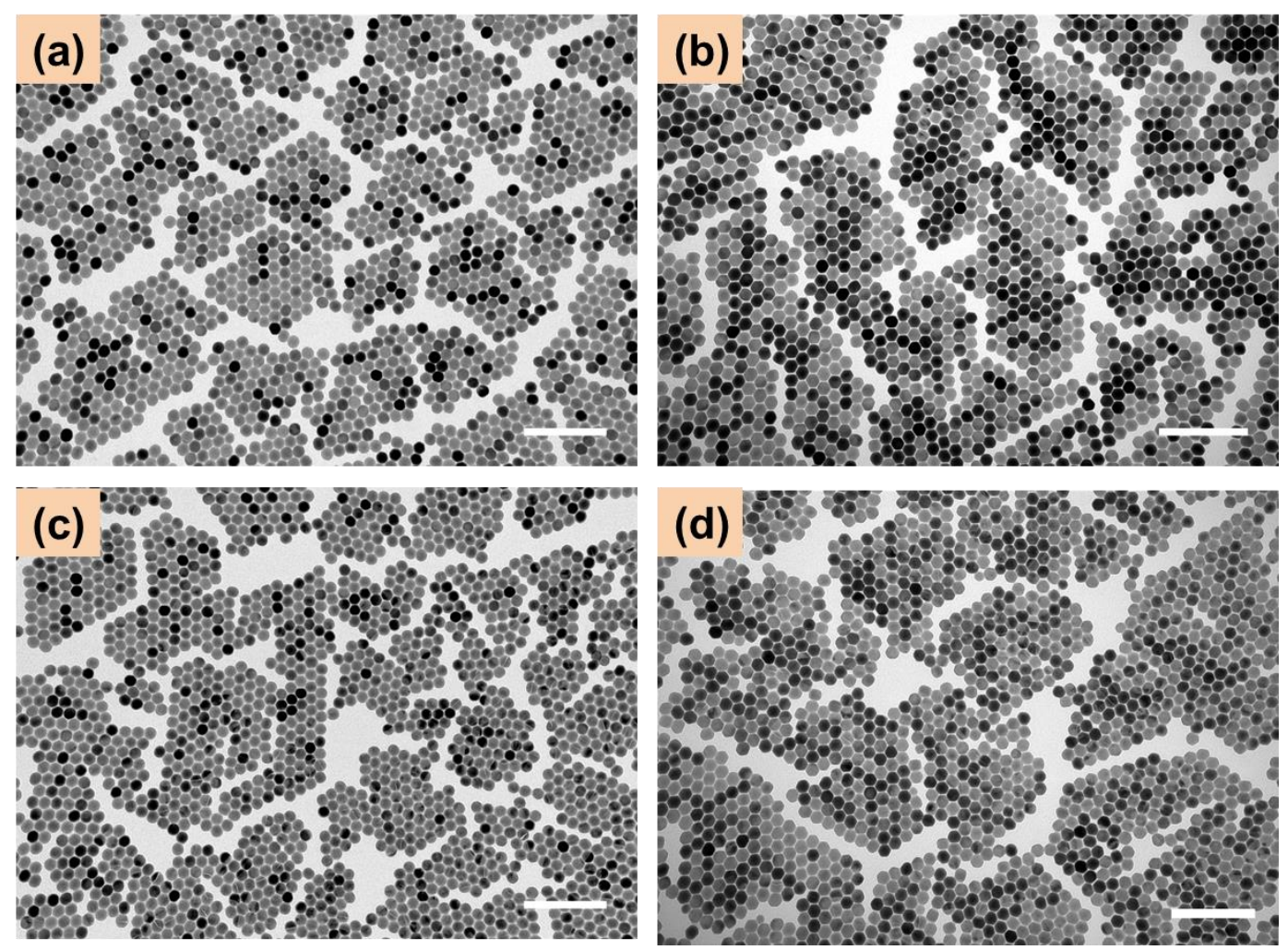

Figure S9. TEM photos of core samples. (a) $\mathrm{NaYF}_{4}: 20 \% \mathrm{Yb}^{3+}, 2 \% \mathrm{Er}^{3+}$ core nanoparticles. (b) $\mathrm{NaYF}_{4}: 30 \% \mathrm{Yb}^{3+}, 2 \%$ $\mathrm{Er}^{3+}$ core nanoparticles. (c) $\mathrm{NaYF}_{4}: 30 \% \mathrm{Yb}^{3+}, 8 \% \mathrm{Er}^{3+}$ core nanoparticles. (d) $\mathrm{NaYF}_{4}: 40 \% \mathrm{Yb}^{3+}, 2 \% \mathrm{Er}^{3+}$ core nanoparticles. Scale bar: $200 \mathrm{~nm}$ 

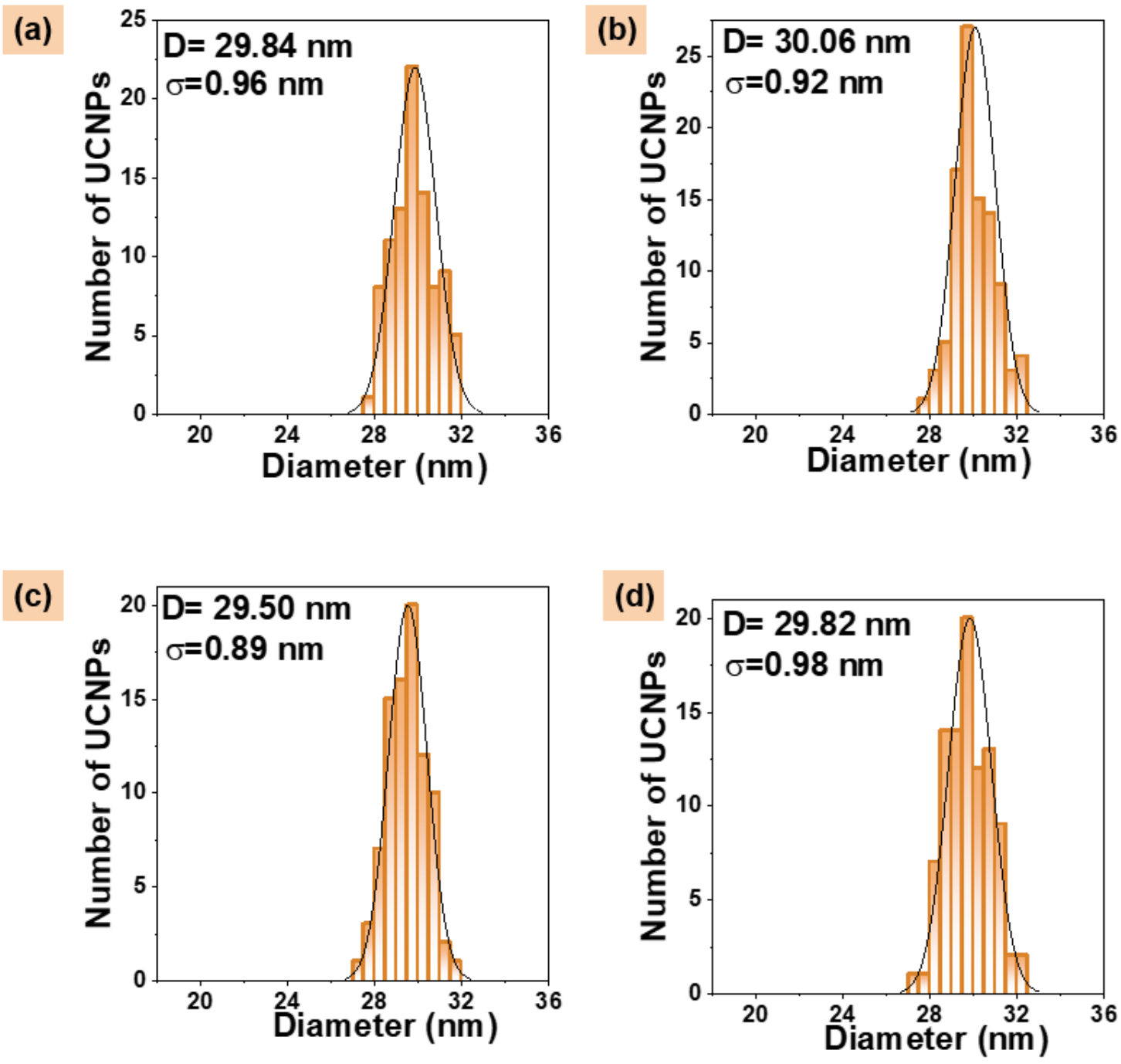

Figure S10. Size distribution of core nanoparticles. (a) $\mathrm{NaYF}_{4}: 20 \% \mathrm{Yb}^{3+}, 2 \% \mathrm{Er}^{3+}$ core nanoparticles. (b) $\mathrm{NaYF}_{4}: 30 \% \mathrm{Yb}^{3+}, 2 \% \mathrm{Er}^{3+}$ core nanoparticles. (c) $\mathrm{NaYF}_{4}: 30 \% \mathrm{Yb}^{3+}, 8 \% \mathrm{Er}^{3+}$ core nanoparticles. (d) $\mathrm{NaYF} 4: 40 \% \mathrm{Yb}^{3+}, 2 \%$ $\mathrm{Er}^{3+}$ core nanoparticles. 

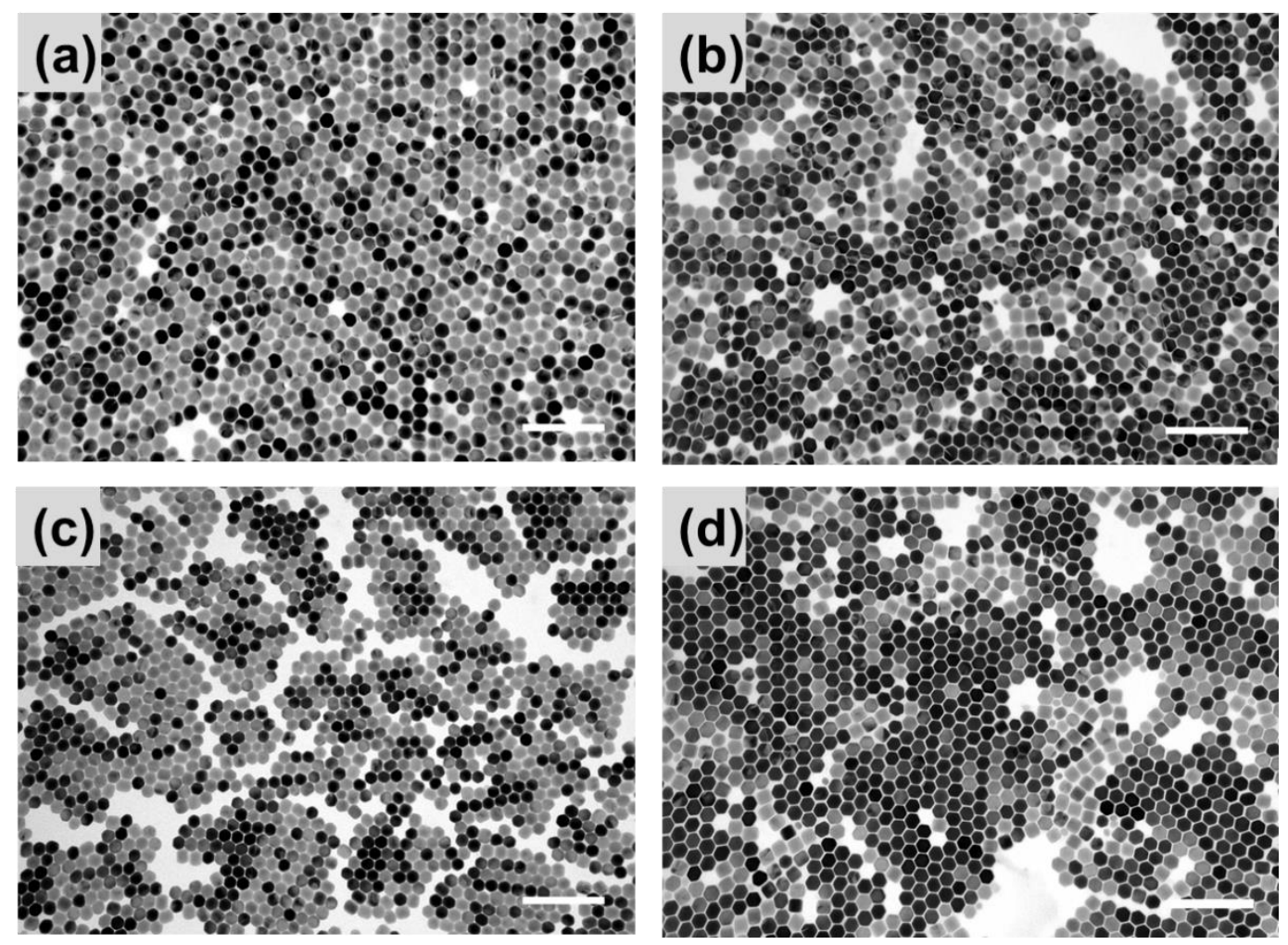

Figure S11. TEM photos of core-shell nanoparticles (a) $\mathrm{NaYF}_{4}: 20 \% \mathrm{Yb}^{3+}, 2 \% \mathrm{Er}^{3+} @ \mathrm{NaYF}_{4}: 5 \% \mathrm{Yb}^{3+}$ nanoparticles. (b) $\mathrm{NaYF}_{4}: 30 \% \mathrm{Yb}^{3+}, 2 \% \mathrm{Er}^{3+} @ \mathrm{NaYF}_{4}: 5 \% \mathrm{Yb}^{3+}$ nanoparticles. (c) $\mathrm{NaYF}_{4}: 30 \% \mathrm{Yb}^{3+}, 8 \% \mathrm{Er}^{3+} @ \mathrm{NaYF}_{4}: 5 \% \mathrm{Yb}^{3+}$ nanoparticles. (d) $\mathrm{NaYF}_{4}: 40 \% \mathrm{Yb}^{3+}, 2 \% \mathrm{Er}^{3+} @ \mathrm{NaYF}_{4}: 5 \% \mathrm{Yb}^{3+}$ nanoparticles. Scale bar: $200 \mathrm{~nm}$ 
(a)

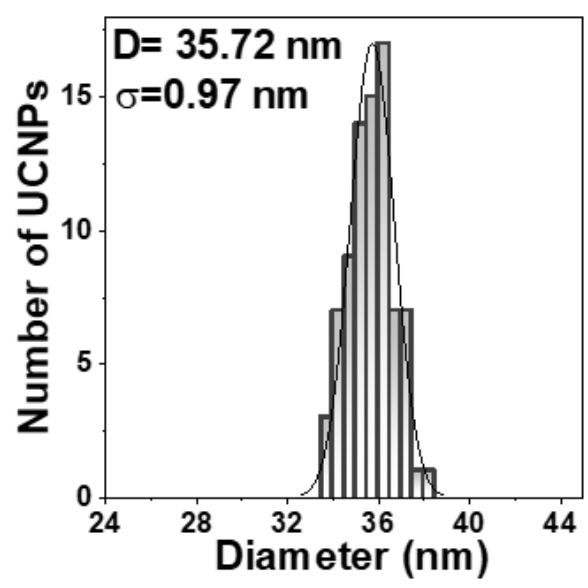

(c)

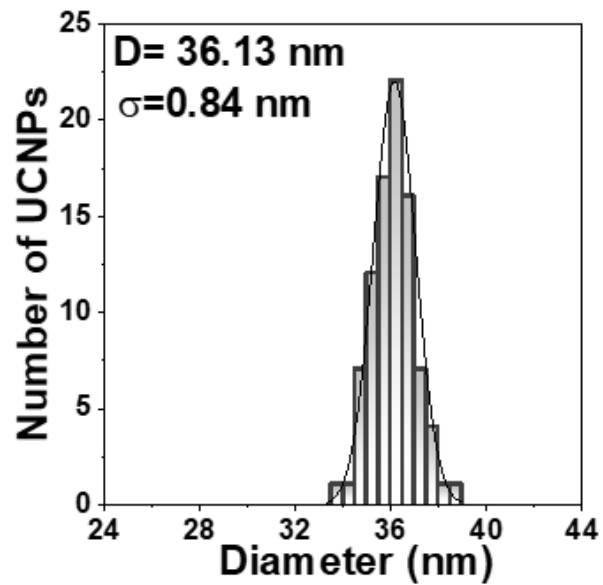

(b)

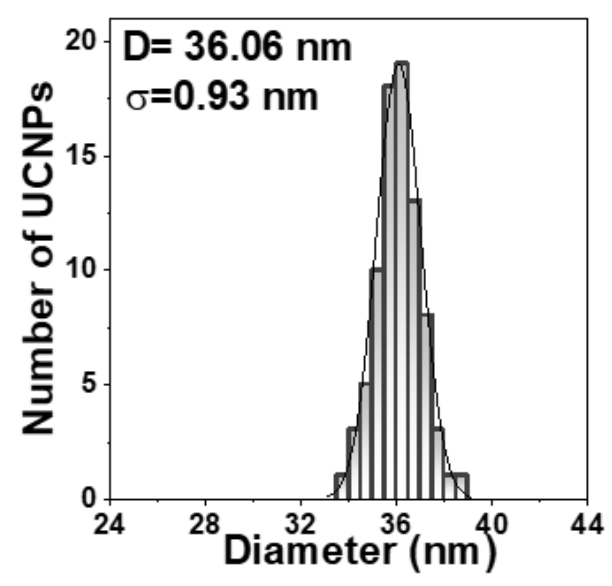

(d)

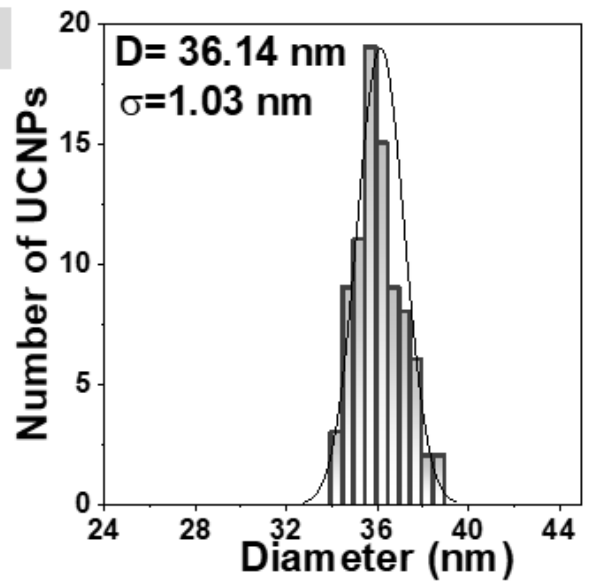

Figure S12. Size distribution of core-shell nanoparticles. (a) $\mathrm{NaYF}_{4}: 20 \% \mathrm{Yb}^{3+}, 2 \% \mathrm{Er}^{3+} @ \mathrm{NaYF}_{4}: 5 \% \mathrm{Yb}^{3+}$ nanoparticles. (b) $\mathrm{NaYF}_{4}: 30 \% \mathrm{Yb}^{3+}, 2 \% \mathrm{Er}^{3+} @ \mathrm{NaYF}_{4}: 5 \% \mathrm{Yb}^{3}$ nanoparticles. (c) $\mathrm{NaYF}_{4}: 30 \% \mathrm{Yb}^{3+}, 8 \% \mathrm{Er}^{3+} @$ $\mathrm{NaYF}_{4}: 5 \% \mathrm{Yb}^{3+}$ nanoparticles. (d) $\mathrm{NaYF}_{4}: 40 \% \mathrm{Yb}^{3+}, 2 \% \mathrm{Er}^{3+} @ \mathrm{NaYF}_{4}: 5 \% \mathrm{Yb}^{3+}$ nanoparticles. 

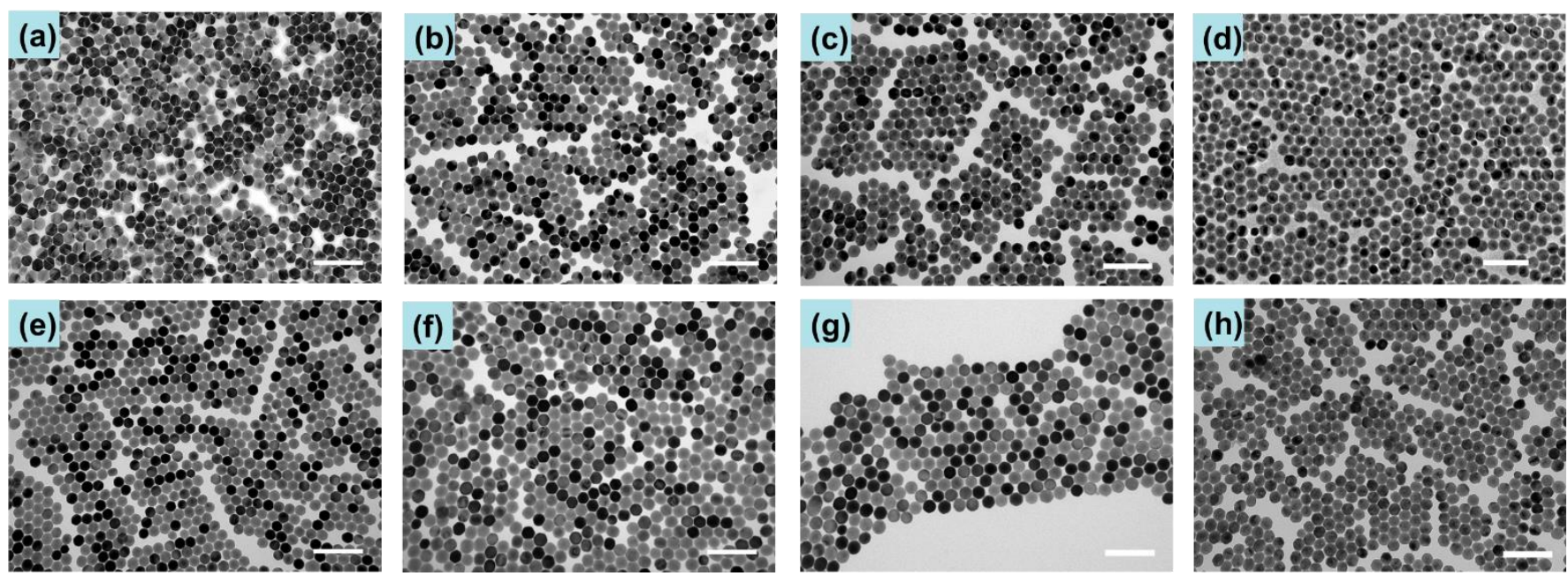

Figure S13. TEM photos of core-shell-shell nanoparticles.

(a) $\mathrm{NaYF}_{4}: 30 \% \mathrm{Yb}^{3+}, 2 \% \mathrm{Er}^{3+} @ \mathrm{NaYF}_{4}: 5 \% \mathrm{Yb}^{3+} @ \mathrm{NaYF}_{4}: 15 \% \mathrm{Yb}^{3+}, 20 \% \mathrm{Nd}^{3+}$ nanoparticles.

(b) $\mathrm{NaYF}_{4}: 30 \% \mathrm{Yb}^{3+}, 8 \% \mathrm{Er}^{3+} @ \mathrm{NaYF}_{4}: 5 \% \mathrm{Yb}^{3+} @ \mathrm{NaYF}_{4}: 15 \% \mathrm{Yb}^{3+}, 20 \% \mathrm{Nd}^{3+}$ nanoparticles.

(c) $\mathrm{NaYF}_{4}: 20 \% \mathrm{Yb}^{3+}, 2 \% \mathrm{Er}^{3+} @ \mathrm{NaYF}_{4}: 5 \% \mathrm{Yb}^{3+} @ \mathrm{NaYF}_{4}: 15 \% \mathrm{Yb}^{3+}, 20 \% \mathrm{Nd}^{3+}$ nanoparticles.

(d) $\mathrm{NaYF}_{4}: 40 \% \mathrm{Yb}^{3+}, 2 \% \mathrm{Er}^{3+} @ \mathrm{NaYF}_{4}: 5 \% \mathrm{Yb}^{3+} @ \mathrm{NaYF}_{4}: 15 \% \mathrm{Yb}^{3+}, 20 \% \mathrm{Nd}^{3+}$ nanoparticles.

(e) $\mathrm{NaYF}_{4}: 30 \% \mathrm{Yb}^{3+}, 2 \% \mathrm{Er}^{3+} @ \mathrm{NaYF}_{4}: 5 \% \mathrm{Yb}^{3+} @ \mathrm{NaYF}_{4}: 5 \% \mathrm{Yb}^{3+}, 20 \% \mathrm{Nd}^{3+}$ nanoparticles.

(f) $\mathrm{NaYF}_{4}: 30 \% \mathrm{Yb}^{3+}, 2 \% \mathrm{Er}^{3+} @ \mathrm{NaYF}_{4}: 5 \% \mathrm{Yb}^{3+} @ \mathrm{NaYF}_{4}: 30 \% \mathrm{Yb}^{3+}, 20 \% \mathrm{Nd}^{3+}$ nanoparticles.

(g) $\mathrm{NaYF}_{4}: 30 \% \mathrm{Yb}^{3+}, 2 \% \mathrm{Er}^{3+} @ \mathrm{NaYF}_{4}: 5 \% \mathrm{Yb}^{3+} @ \mathrm{NaYF}_{4}: 15 \% \mathrm{Yb}^{3+}, 40 \% \mathrm{Nd}^{3+}$ nanoparticles.

(h) $\mathrm{NaYF}_{4}: 30 \% \mathrm{Yb}^{3+}, 2 \% \mathrm{Er}^{3+} @ \mathrm{NaYF}_{4}: 5 \% \mathrm{Yb}^{3+} @ \mathrm{NaYF}_{4}: 15 \% \mathrm{Yb}^{3+}, 60 \% \mathrm{Nd}^{3+}$ nanoparticles. 
(a)

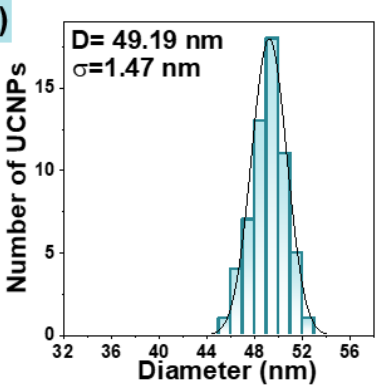

(e) ${ }^{25} \mathrm{D}=49.55 \mathrm{~nm}$

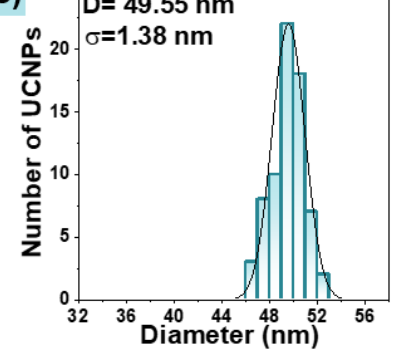

(b) 2

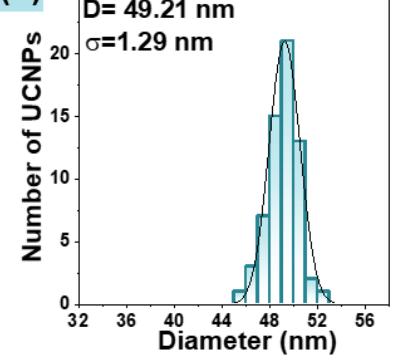

(f) ${ }^{25} \mathrm{D}=50.29 \mathrm{~nm}$

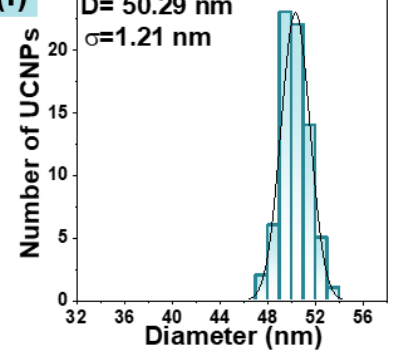

(c) 25

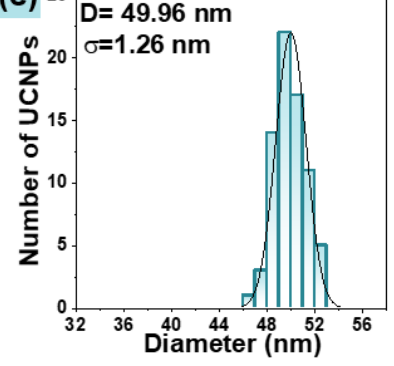

(g)

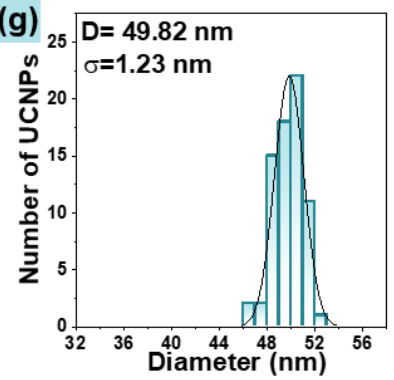

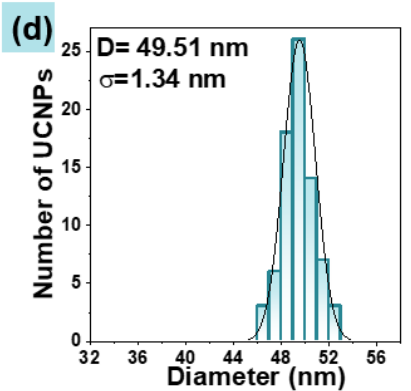

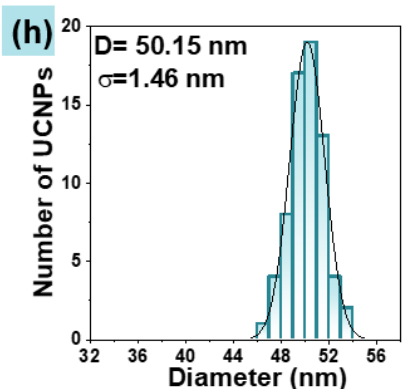

Figure S14. Size distribution of core-shell-shell nanoparticles.

(a) $\mathrm{NaYF}_{4}: 30 \% \mathrm{Yb}^{3+}, 2 \% \mathrm{Er}^{3+} @ \mathrm{NaYF}_{4}: 5 \% \mathrm{Yb}^{3+} @ \mathrm{NaYF}_{4}: 15 \% \mathrm{Yb}^{3+}, 20 \% \mathrm{Nd}^{3+}$ nanoparticles.

(b) $\mathrm{NaYF}_{4}: 30 \% \mathrm{Yb}^{3+}, 8 \% \mathrm{Er}^{3+} @ \mathrm{NaYF}_{4}: 5 \% \mathrm{Yb}^{3+} @ \mathrm{NaYF}_{4}: 15 \% \mathrm{Yb}^{3+}, 20 \% \mathrm{Nd}^{3+}$ nanoparticles.

(c) $\mathrm{NaYF}_{4}: 20 \% \mathrm{Yb}^{3+}, 2 \% \mathrm{Er}^{3+} @ \mathrm{NaYF}_{4}: 5 \% \mathrm{Yb}^{3+} @ \mathrm{NaYF}_{4}: 15 \% \mathrm{Yb}^{3+}, 20 \% \mathrm{Nd}^{3+}$ nanoparticles.

(d) $\mathrm{NaYF}_{4}: 40 \% \mathrm{Yb}^{3+}, 2 \% \mathrm{Er}^{3+} @ \mathrm{NaYF}_{4}: 5 \% \mathrm{Yb}^{3+} @ \mathrm{NaYF}_{4}: 15 \% \mathrm{Yb}^{3+}, 20 \% \mathrm{Nd}^{3+}$ nanoparticles.

(e) $\mathrm{NaYF}_{4}: 30 \% \mathrm{Yb}^{3+}, 2 \% \mathrm{Er}^{3+} @ \mathrm{NaYF}_{4}: 5 \% \mathrm{Yb}^{3+} @ \mathrm{NaYF}_{4}: 5 \% \mathrm{Yb}^{3+}, 20 \% \mathrm{Nd}^{3+}$ nanoparticles.

(f) $\mathrm{NaYF}_{4}: 30 \% \mathrm{Yb}^{3+}, 2 \% \mathrm{Er}^{3+} @ \mathrm{NaYF}_{4}: 5 \% \mathrm{Yb}^{3+} @ \mathrm{NaYF}_{4}: 30 \% \mathrm{Yb}^{3+}, 20 \% \mathrm{Nd}^{3+}$ nanoparticles.

(g) $\mathrm{NaYF}_{4}: 30 \% \mathrm{Yb}^{3+}, 2 \% \mathrm{Er}^{3+} @ \mathrm{NaYF}_{4}: 5 \% \mathrm{Yb}^{3+} @ \mathrm{NaYF}_{4}: 15 \% \mathrm{Yb}^{3+}, 40 \% \mathrm{Nd}^{3+}$ nanoparticles.

(h) $\mathrm{NaYF}_{4}: 30 \% \mathrm{Yb}^{3+}, 2 \% \mathrm{Er}^{3+} @ \mathrm{NaYF}_{4}: 5 \% \mathrm{Yb}^{3+} @ \mathrm{NaYF}_{4}: 15 \% \mathrm{Yb}^{3+}, 60 \% \mathrm{Nd}^{3+}$ nanoparticles. 

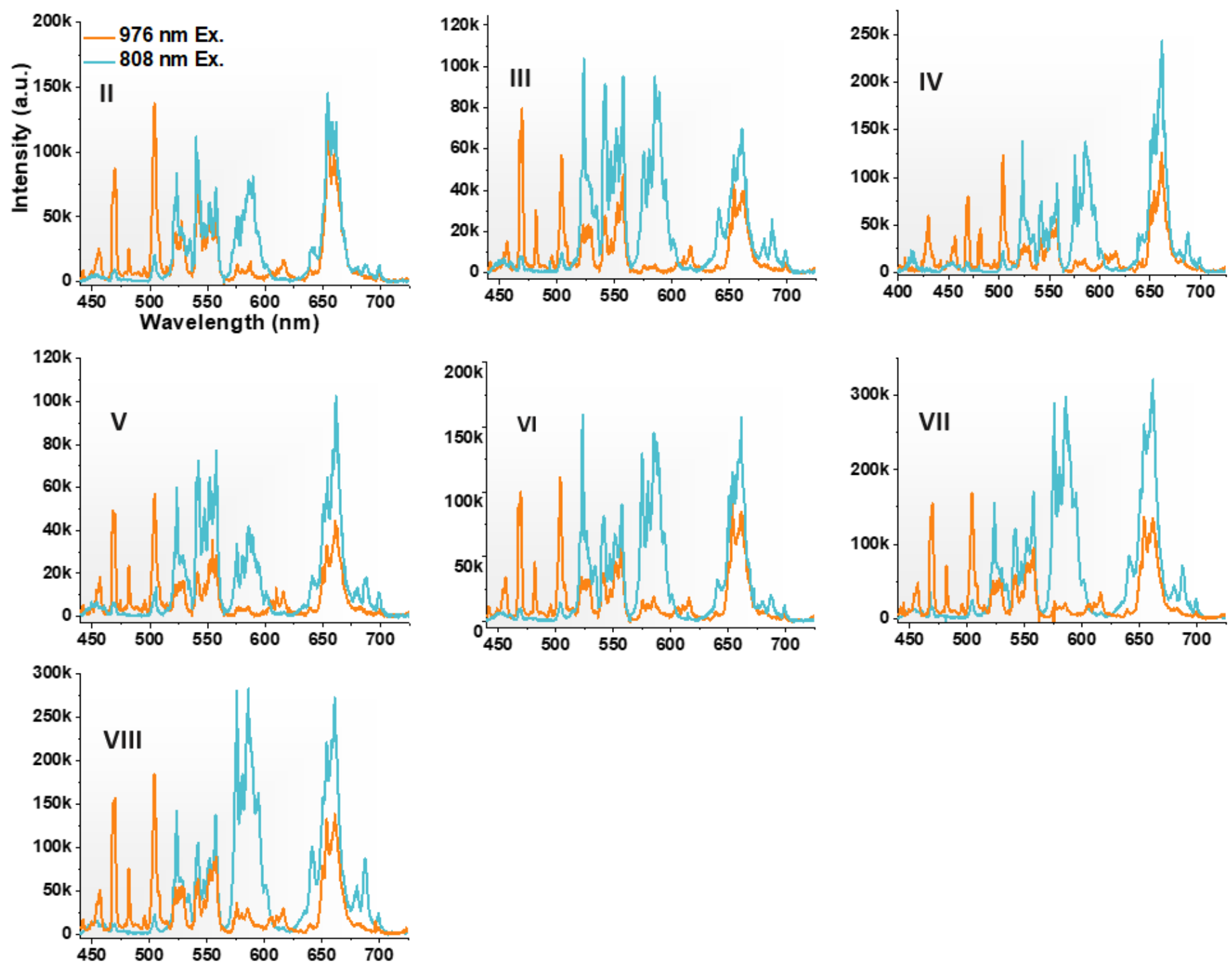

Figure S15. (a) Upconversion emission spectra of sample II (a), sample III (b), sample IV (c), sample V (d), sample VI (e), sample VII (f), sample VIII (g) under $976 \mathrm{~nm}$ (orange) and $808 \mathrm{~nm}$ (blue) excitation at $7.6 \mathrm{MW} / \mathrm{cm}^{2}$. 


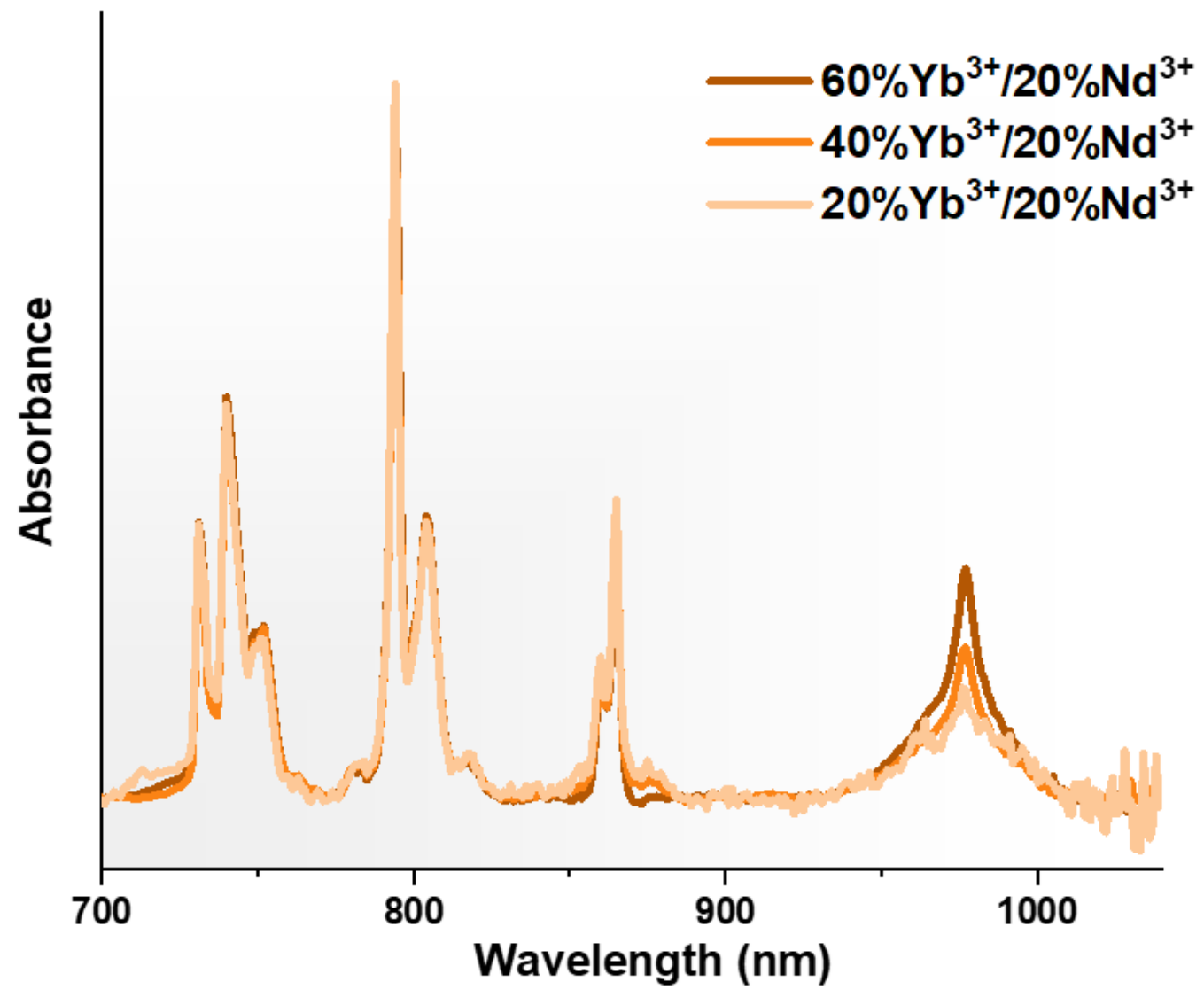

Figure S16. The absorption spectra of $\mathrm{Yb}^{3+}-\mathrm{Nd}^{3+}$ co-doped nanoparticles with different $\mathrm{Yb}^{3+}(20 \%, 40 \%, 60 \%)$ concentrations, which are normalized according to the absorbance at $795 \mathrm{~nm}$. 
a

$\mathrm{Yb}^{3+}$ Con. in Sensitization Layer

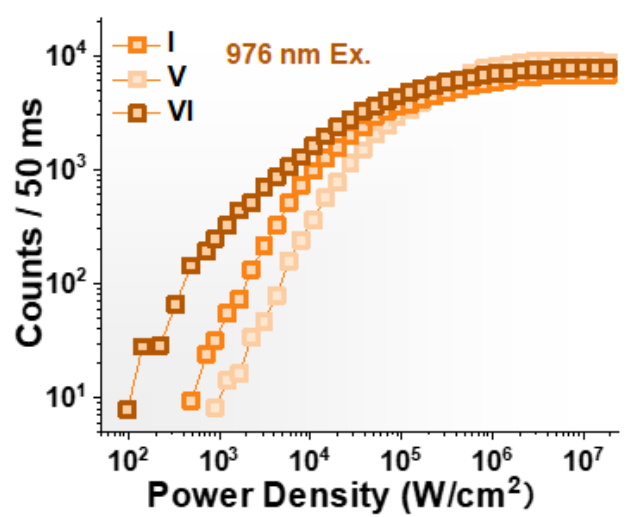

b

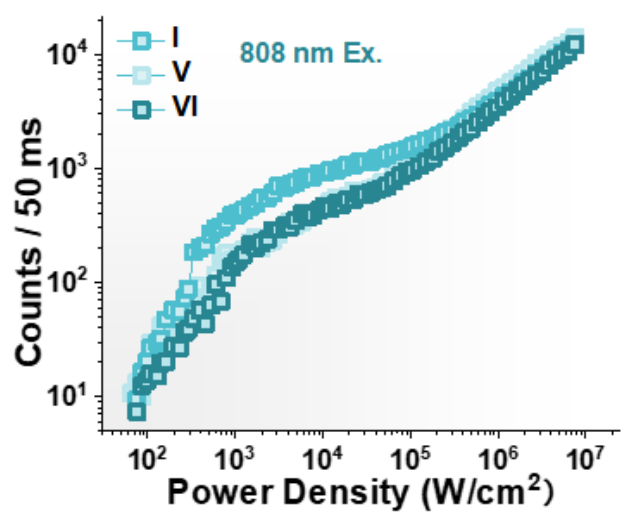

C

$\mathrm{Nd}^{3+}$ Con. in Sensitization Layer

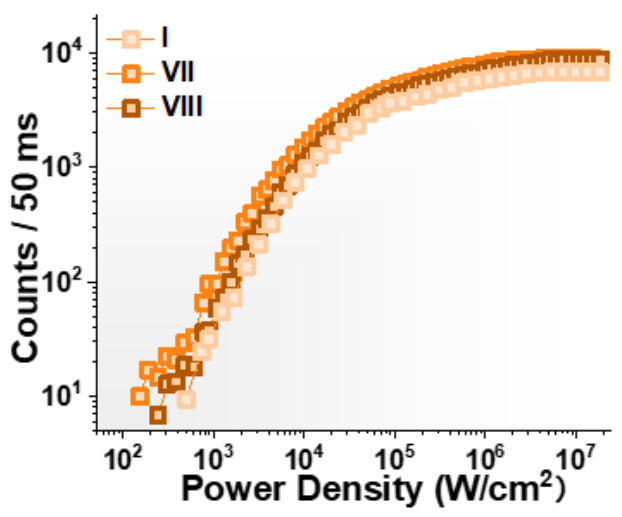

d

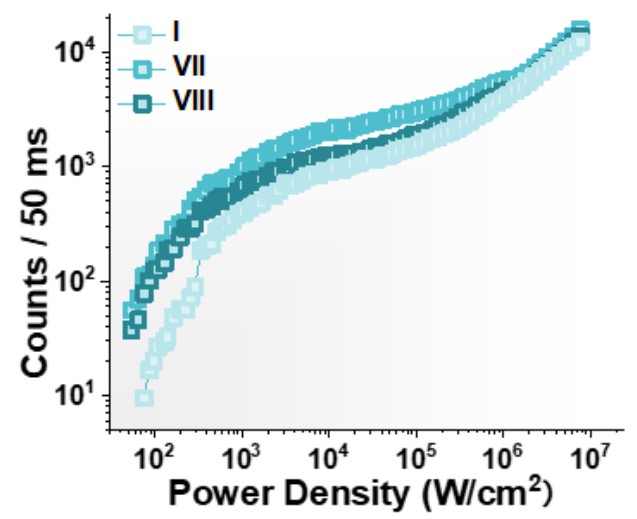

Figure S17. The comparison of the emission intensity as the function of power density under $976 \mathrm{~nm}$ (a) and $808 \mathrm{~nm}$ (b) excitations for sample I, V and VI. The comparison of the emission intensity as the function of power density under $976 \mathrm{~nm}$ (c) and $808 \mathrm{~nm}$ (d) excitations for sample I, VII and VIII. 


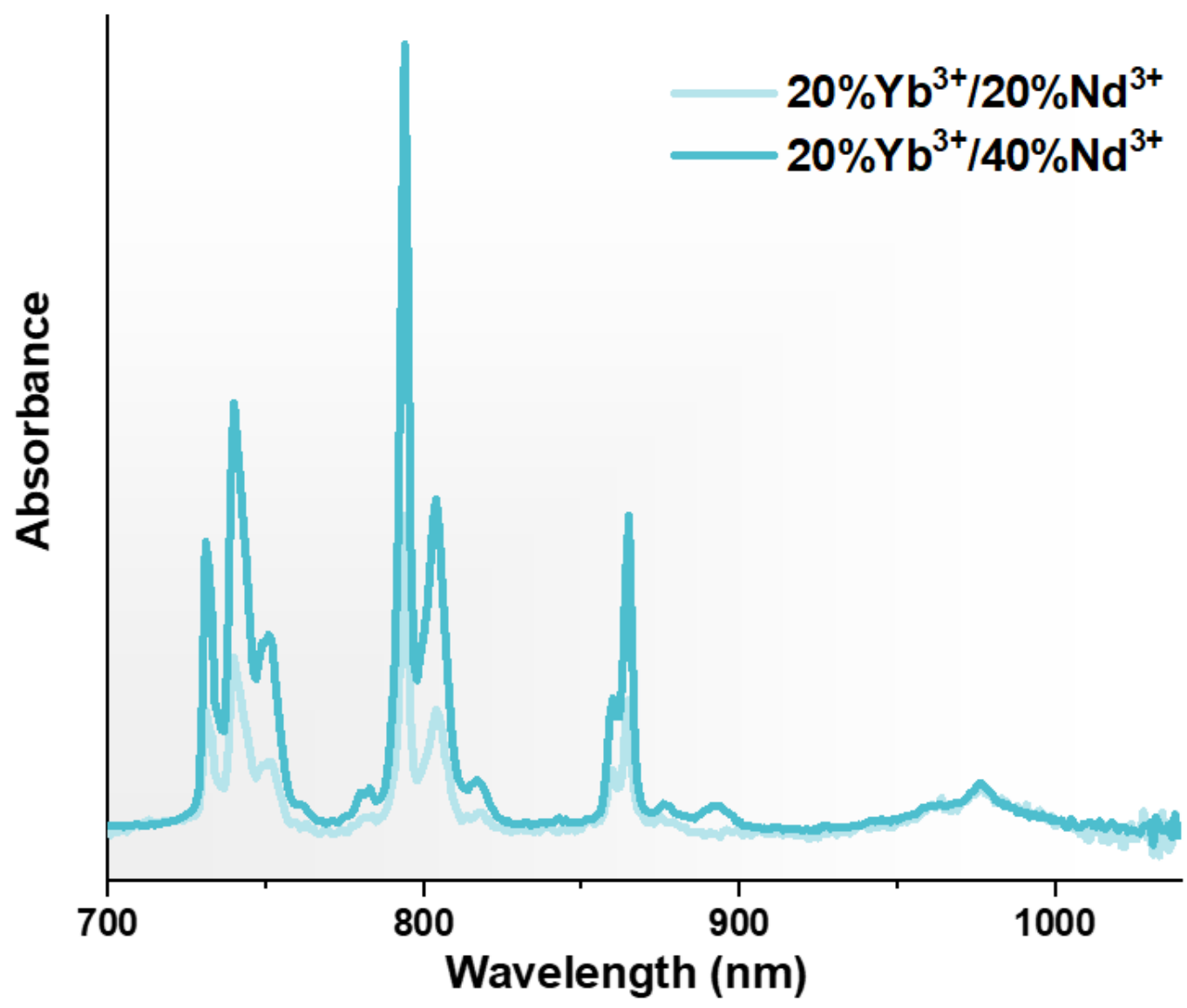

Figure S18. The absorption spectra of $\mathrm{Yb}^{3+}-\mathrm{Nd}^{3+}$ co-doped nanoparticles with different $\mathrm{Nd}^{3+}(20 \%, 40 \%)$ concentrations, which are normalized according to the absorbance at $976 \mathrm{~nm}$. 
a

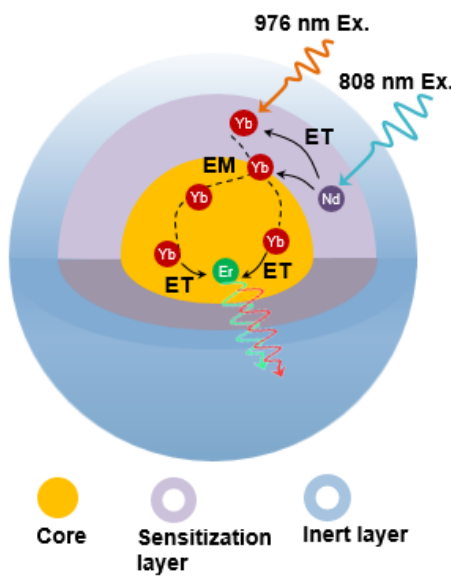

b

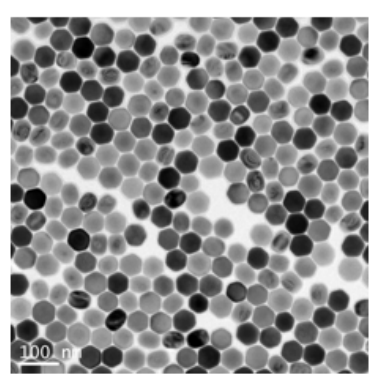

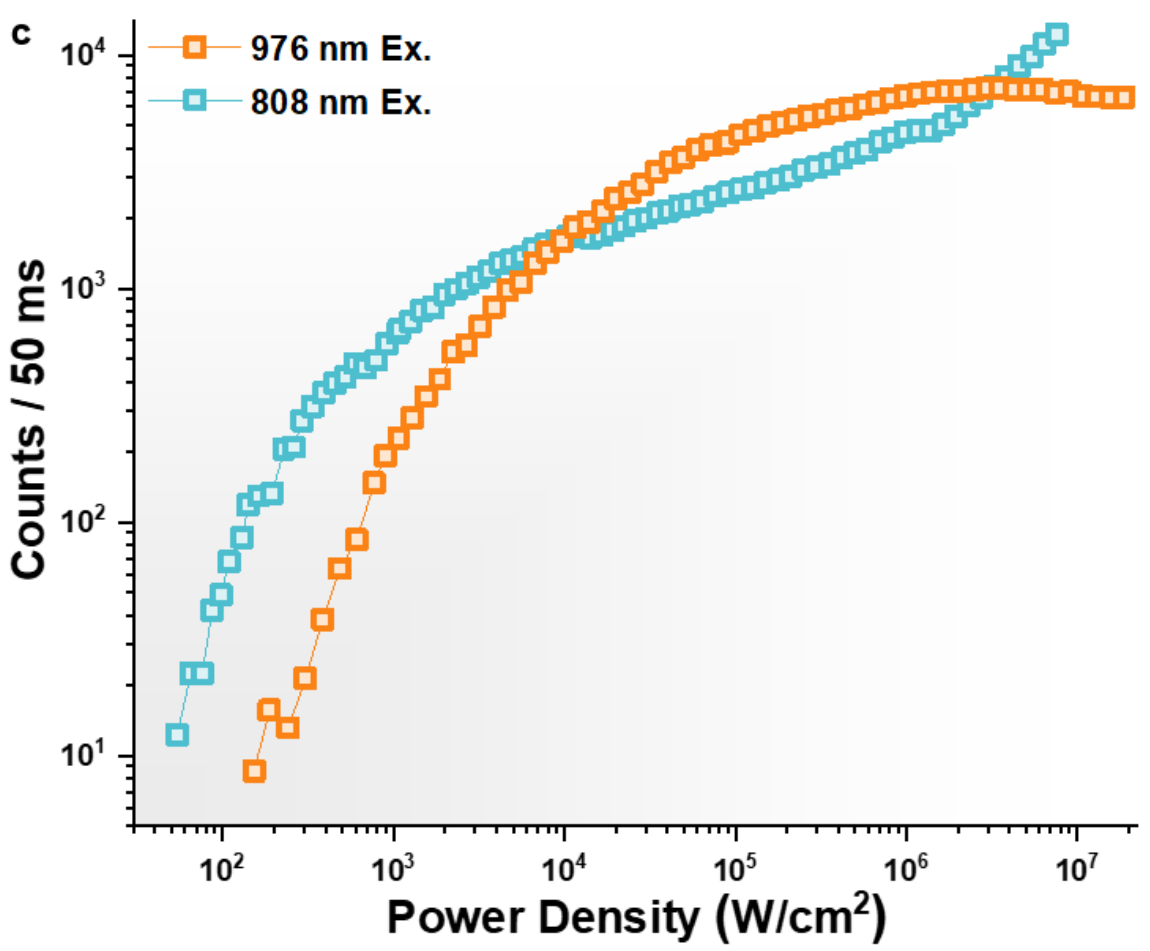

Figure S19. (a) Schematic illustration of the three-layer core multi-shell nanostructure and energy transfer processes under the excitations at $976 \mathrm{~nm}$ and $808 \mathrm{~nm}$. (b) TEM image of the three-layer nanoparticles $\left(\mathrm{NaYF}_{4}: 30 \% \mathrm{Yb}: 2 \% \operatorname{Er} @\right.$ $\left.\mathrm{NaYF}_{4}: 15 \% \mathrm{Yb}: 20 \% \mathrm{Nd} @ \mathrm{NaYF}_{4}\right)(\mathrm{c})$ The power-dependent intensity curves of single nanoparticle under $976 \mathrm{~nm}$ and $808 \mathrm{~nm}$ excitations. 